Supporting Information for

\title{
Chiral DHIP-based metal-organic frameworks for enantioselective recognition and separation
}

\author{
Jie Zhang, ${ }^{\dagger}$ Zijian Li, ${ }^{\dagger}$ Wei Gong, ${ }^{\dagger}$ Xing Han,${ }^{\dagger}$ Yan $\mathrm{Liu}^{*}{ }^{\dagger}$ and Yong Cui ${ }^{*}, \dagger, \star$ \\ ${ }^{\dagger}$ School of Chemistry and Chemical Engineering and State Key Laboratory of Metal Matrix \\ Composites, Shanghai Jiao Tong University, Shanghai 200240, China; \\ ${ }^{\ddagger}$ Collaborative Innovation Center of Chemical Science and Engineering, Tianjin 300072, China \\ E-mail: yongcui@sjtu.edu.cn, liuy@sjtu.edu.cn
}

\section{Table of Content}

1. Materials and general procedures

2. Synthesis

3. Experimental procedure for adsorption separation

4.Table S1. Crystal data and structure refinement for 1 and 2

5. Table S2-S3. Selected bond lengths $[\AA ̊]$ and angles $\left[{ }^{\circ}\right]$ for 1 and 2

6. Figures S1-S8. Additional X-ray crystallographic structures

7. Figure S9. ESI-MS spectra

8. Figure S10. PXRD patterns

9. Figure S11. TGA curves

10. Figure S12. Solid-state CD spectra

11. Figure $\mathrm{S} 13$. The $\mathrm{CO}_{2}$ adsorption isotherms

12. NMR spectra

13. HPLC results

14. Kinetics of adsorption

15. Figure S14. FT-IR spectra 


\section{Materials and general procedures}

All of the chemicals are commercial available, and used without further purification. The IR (KBr pellet) spectra were recorded $\left(400-4000 \mathrm{~cm}^{-1}\right.$ region) on a Nicolet Magna 750 FT-IR spectrometer. CD spectra were recorded on a J-800 spectropolarimeter (Jasco, Japan). Thermogravimetric analyses (TGA) were carried out in an air atmosphere with a heating rate of $10{ }^{\circ} \mathrm{C} / \mathrm{min}$ on a STA449C integration thermal analyzer. Powder X-ray diffraction (PXRD) data were collected on a DMAX2500 diffractometer using $\mathrm{Cu} \mathrm{K} \alpha$ radiation. The calculated PXRD patterns were produced using the SHELXTL-XPOW program and single crystal reflection data. NMR experiments were carried out on a MERCURY plus 400 spectrometer operating at resonance frequencies of $400 \mathrm{MHz}$. ESI-MS spectra were recorded on a Finnigan LCQ mass spectrometer using dichloromethane-methanol as mobile phase. ICP-OES was performed on Optima 7300DV ICP-OES (Perkin Elmer Coporation, USA). Analytical high performance liquid chromatography (HPLC) was performed on YL-9100 HPLC. Analytical CHIRALCEL OD-Hcolumn from Daicel was used. The $\mathrm{CO}_{2}$ adsorption isotherms were recorded at $273 \mathrm{~K}$ by using a micromeritics ASAP 2020 surface area and porosity analyzer. Before the adsorption measurement, the samples were activated at $80^{\circ} \mathrm{C}$ under vacuum $\left(<10^{-3}\right.$ torr $)$ for $4 \mathrm{~h}$ 。

X-ray Crystallography. Single-crystal XRD data for $\mathbf{1}$ and $\mathbf{2}$ were collected on a Bruker SMART Apex II CCD-based X-ray diffractometer with Mo-K $\alpha$ radiation $(\lambda=$ $0.71073 \AA$ ) at 298 or $259 \mathrm{~K}$. The empirical absorption correction was applied by using the SADABS program (G. M. Sheldrick, SADABS, program for empirical absorption correction of area detector data; University of Göttingen, Göttingen, Germany, 1996).The structure was solved using direct method, and refined by full-matrix least-squares on $\mathrm{F}^{2}$ (G. M. Sheldrick, SHELXTL97, program for crystal structure refinement, University of Göttingen, Germany, 1997). All non-H atoms were refined anisotropically. Contributions to scattering due to these highly disordered solvent molecules were removed using the SQUEEZE routine of PLATON;structures were then refined again using the data generated. Crystal data and details of the data collection are given in Table S1, while the selected bond distances and angles are presented in Table S2-S3.

\section{Synthesis}

\subsection{Synthesis of a}

A $25-\mathrm{mL}$ pressure tube charged with 2,5-dibromopyridine $(237 \mathrm{mg}, 1.00 \mathrm{mmol}$ ), (R)-2-amino-2-(4-bromophenyl)ethanol $\quad(216 \mathrm{mg}, 1.00 \mathrm{mmol}), \quad i$ - $\operatorname{Pr}_{2} \mathrm{NEt} \quad(150 \mathrm{mg}$, 
$1.16 \mathrm{mmol}$ ) and a stir bar was flushed with nitrogen several times, stoppered and heated at $165^{\circ} \mathrm{C}$ for 4 days. The tube was allowed to cool to room temperature, and the contents was diluted with a small amount of $\mathrm{CH}_{2} \mathrm{Cl}_{2}$ and chromatographed (2:1 hexanes-EtOAc) to afford product as a white solid (186mg, 50\% yield).

${ }^{1} \mathrm{H}$ NMR (400 MHz, $\left.\mathrm{CDCl}_{3}\right): \delta 8.00(\mathrm{~d}, J=2.1 \mathrm{~Hz}, 1 \mathrm{H}), 7.50-7.36(\mathrm{~m}, 3 \mathrm{H})$, 7.24-7.15 (m, 2H), $6.17(\mathrm{~d}, J=8.8 \mathrm{~Hz}, 1 \mathrm{H}), 5.56$ (d, $J=5.6 \mathrm{~Hz}, 1 \mathrm{H}), 4.69$ (dd, $J=9.6$, $6.3 \mathrm{~Hz}, 1 \mathrm{H}), 3.92(\mathrm{dd}, J=11.1,3.7 \mathrm{~Hz}, 1 \mathrm{H}), 3.79(\mathrm{dd}, J=11.1,6.8 \mathrm{~Hz}, 1 \mathrm{H}) .{ }^{13} \mathrm{C} \mathrm{NMR}$ (400 MHz, $\left.\mathrm{CDCl}_{3}\right): \delta 156.85,148.46,140.39,139.02,132.14,128.65,121.80,109.38$, 107.98, 66.84, 58.29. ESI-MS: m/z $372.9\left(\right.$ Calcd m/z 371.9 for $\left.[\mathrm{L}+\mathrm{H}]^{+}\right)$.

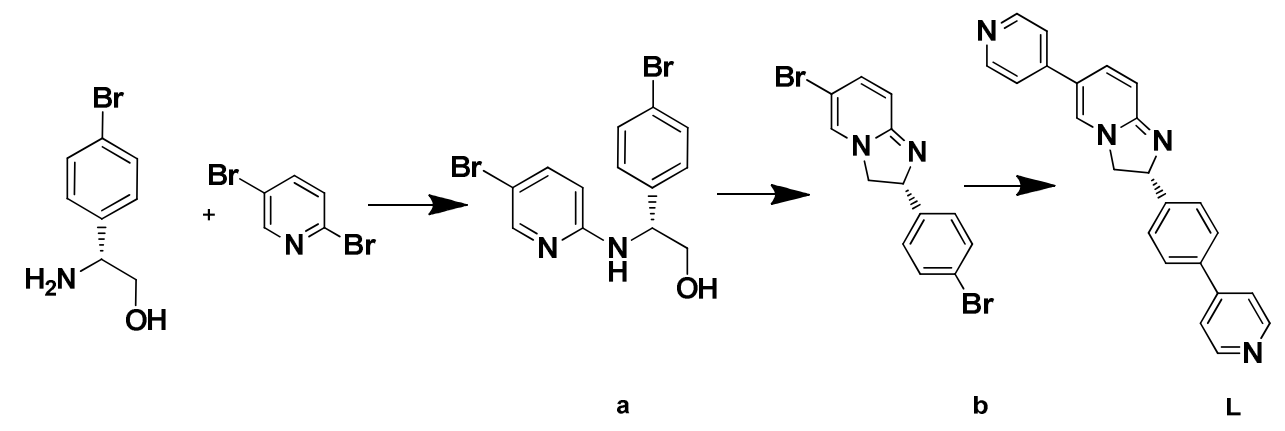

\subsection{Synthesis of $b$}

To a solution of a $(372 \mathrm{mg}, 1.00 \mathrm{mmol})$ in $6 \mathrm{~mL} \mathrm{CHCl} 3$ was added $\mathrm{SOCl}_{2}$ $(0.150 \mathrm{~mL}, 2.06 \mathrm{mmol})$ dropwise at room temperature, then heated to reflux. After $1 \mathrm{~h}$, the flask was allowed to cool and treated cautiously with 2-3 drops of $\mathrm{MeOH}$ (vigorous gas evolution!), then heated again for $5 \mathrm{~min}$. The solvent was removed under reduced pressure, and the residue was extracted with warm water. The aqueous extract was filtered from gummy particles through a cotton plug, basified with concentrated $\mathrm{NaOH}$ and extracted with benzene. The benzene solution was dried briefly over $\mathrm{Na}_{2} \mathrm{SO}_{4}$ and then heated under reflux in an oil bath at $80-85^{\circ} \mathrm{C}$. After 2 hours, the solution was evaporated to give bright-yellow crystalline product (248mg, $70 \%$ yield).

${ }^{1} \mathrm{H}$ NMR (400 MHz, $\left.\mathrm{CDCl}_{3}\right): \delta$ 7.53-7.41 (m, 2H), $7.19(\mathrm{~d}, J=8.4 \mathrm{~Hz}, 2 \mathrm{H}), 7.06$ $(\mathrm{d}, J=1.9 \mathrm{~Hz}, 1 \mathrm{H}), 6.87(\mathrm{dd}, J=9.9,2.1 \mathrm{~Hz}, 1 \mathrm{H}), 6.37(\mathrm{~d}, J=9.9 \mathrm{~Hz}, 1 \mathrm{H}), 5.21(\mathrm{dd}$, $J=11.5,9.0 \mathrm{~Hz}, 1 \mathrm{H}), 4.34(\mathrm{t}, J=11.1 \mathrm{~Hz}, 1 \mathrm{H}), 3.79(\mathrm{dd}, J=10.7,9.0 \mathrm{~Hz}, 1 \mathrm{H}) .{ }^{13} \mathrm{C}$ NMR (400 MHz, CDC13): $\delta$ 156.75, 143.21, 140.29, 133.62, 131.99, 128.53, 121.42,

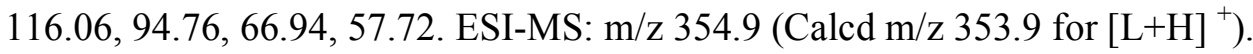

\subsection{Synthesis of $L$}


A mixture of $\mathbf{b}(1.77 \mathrm{~g}, 5 \mathrm{mmol})$, 4-pyridylboronic acid $(1.54 \mathrm{~g}, 12.45 \mathrm{mmol})$, $\mathrm{Pd}\left(\mathrm{PPh}_{3}\right)_{4}(115.6 \mathrm{mg}, 0.10 \mathrm{mmol})$, and $\mathrm{K}_{3} \mathrm{PO}_{4}(6.0 \mathrm{~g}, 41.5 \mathrm{mmol})$ in dioxane $/ \mathrm{H}_{2} \mathrm{O}(3: 1$ $\mathrm{v} / \mathrm{v}, 60 \mathrm{~mL}$ ) was degassed for $10 \mathrm{~min}$, and then the suspension was stirred at $100^{\circ} \mathrm{C}$ under $\mathrm{N}_{2}$ for 1 day. After cooling to room temperature, the mixture was concentrated and acidly with hydrochloric acid, then the water phase was washed with EtOAc, then basicity with $\mathrm{NaOH}$. After filtration, the filter cake was got as the crud product, which was purified by column chromatography on silica gel $(\mathrm{DCM} / \mathrm{MeOH}, 10: 1 \mathrm{v} / \mathrm{v})$ to afford pure $\mathbf{L}$ as a yellow solid (1.14g, 65\% yield).

${ }^{1} \mathrm{H}$ NMR (400 MHz, $\left.\mathrm{CDCl}_{3}\right): \delta 8.63(\mathrm{dd}, J=4.5,1.7 \mathrm{~Hz}, 2 \mathrm{H}), 8.54$ (dd, $J=4.5$, $1.7 \mathrm{~Hz}, 2 \mathrm{H}), 7.67-7.58(\mathrm{~m}, 2 \mathrm{H}), 7.52-7.35(\mathrm{~m}, 5 \mathrm{H}), 7.26(\mathrm{~m}, 3 \mathrm{H}), 6.66-6.57(\mathrm{~m}, 1 \mathrm{H})$, $5.39(\mathrm{dd}, J=11.2,8.6 \mathrm{~Hz}, 1 \mathrm{H}), 4.52(\mathrm{t}, J=11.2 \mathrm{~Hz}, 1 \mathrm{H}), 4.00(\mathrm{dd}, J=11.2,8.6 \mathrm{~Hz}$, $1 \mathrm{H}) .{ }^{13} \mathrm{C}$ NMR (400 MHz, $\left.\mathrm{CD}_{3} \mathrm{OD}\right): \delta 157.94,149.41,149.32,148.96,145.66,145.08$, 136.74, 136.53, 134.26, 127.49, 127.28, 121.79, 119.07, 114.54, 113.14, 66.16, 57.41. ESI-MS: m/z $351.1\left(\right.$ Calcd m/z 350.1 for $\left.[\mathrm{L}+\mathrm{H}]^{+}\right)$.

\subsection{Synthesis of MOF 1}

A mixture of $\mathrm{Zn}\left(\mathrm{NO}_{3}\right)_{2} \cdot 6 \mathrm{H}_{2} \mathrm{O}$ (23.8mg, $\left.0.08 \mathrm{mmol}\right), \mathbf{L}$ (14mg, $0.04 \mathrm{mmol}$ ), $\mathrm{H}_{2} \mathrm{BDC}$ (6.6mg, 0.04mmol), MeOH (1 mL), $\mathrm{CH}_{3} \mathrm{CN}(0.5 \mathrm{~mL})$, DMF (0.5mL) was sealed in a $10 \mathrm{~mL}$ vial with a screw cap and heated at $80{ }^{\circ} \mathrm{C}$ for 5 days. The mixture was then cooled to room temperature and yellow block-like crystals were obtained, washed with methanol and dried in air. Yield: $78 \%$. Calcd for $\mathrm{C}_{70} \mathrm{H}_{54} \mathrm{~N}_{8} \mathrm{O}_{15} \mathrm{Zn}_{3}: \mathrm{C}$, 58.25; H, 3.77; N, 7.76; Found: C, 57.32; H, 3.70; N, 7.68. FTIR (KBr pellet, $\left.v / \mathrm{cm}^{-1}\right)$ : 3434(s), 2923(m), 2853(w), 1653(s), 1614(s), 1591(m), 1567(m), 1509(m), 1373(s), 1295(w), 1265(w), 1244(w), 1145(w), 1073(w), 1043(w), 1017(w), 925(w), 818(s), 747(s), 633(w), 616(w), 521(m).

\subsection{Synthesis of MOF 2}

A mixture of $\mathrm{CdI}_{2}(29.4 \mathrm{mg}, 0.08 \mathrm{mmol}), \mathbf{L}(14 \mathrm{mg}, 0.04 \mathrm{mmol}), \mathrm{H}_{2} \mathrm{BDC}(6.6 \mathrm{mg}$, $0.04 \mathrm{mmol}), \mathrm{MeOH}(1 \mathrm{~mL}), \mathrm{EtOH}(0.5 \mathrm{~mL}), \mathrm{DMF}(0.5 \mathrm{~mL})$ was sealed in a $10 \mathrm{~mL}$ vial with a screw cap and heated at $80{ }^{\circ} \mathrm{C}$ for 5 days. The mixture was then cooled to room temperature and yellow block-like crystals were obtained, washed with methanol and dried in air. Yield: $68 \%$. Calcd for $\mathrm{C}_{70} \mathrm{H}_{54} \mathrm{Cd}_{3} \mathrm{~N}_{8} \mathrm{O}_{15}: \mathrm{C}, 53.06 ; \mathrm{H}, 3.44$;

$\mathrm{N}, 7.07$; Found: C, 52.02; H, 3.40; N, 6.94. FTIR (KBr pellet, $\left.v / \mathrm{cm}^{-1}\right): 3434(\mathrm{~s})$, 2924(w), 2852(w), 1706(w), 1651(m), 1613(m), 1562(m), 1506(m), 1380(m), 1291(w), 1242(m), 1143(m), 1073(w), 1042(w), 1015(w), 958(s), 884(w), 817(s), 750(s), 633(w), 565(w), 525(w). 


\section{Experimental procedure for adsorption separation}

\subsection{Adsorption separation}

Evacuated sample of 1 (30 mg) and sulfoxides in $\mathrm{MeOH}$ were mixed together in a sealed vial at $-10{ }^{\circ} \mathrm{C}$ for $12 \mathrm{~h}$. The solid sample was filtered, washed with $\mathrm{MeOH}$, and then soaked in diethyl ether to obtain the encapsulated guests. Optical purity of the encapsulated sulfoxides was analyzed by HPLC on a ChiralCel OD-H column.

\subsection{The procedure for recycled experiments}

After adsorption separation experiment, the recovered 1 was soaked in fresh diethyl ether for three times and then evacuated at $80{ }^{\circ} \mathrm{C}$ for $4 \mathrm{~h}$. The recycled 1 and sulphoxides in $\mathrm{MeOH}$ were mixed together in a sealed vial at $-10^{\circ} \mathrm{C}$ for 12 hours. The following workup is identical to section 3.1. The other two recycled experiments were performed in a similar procedure.

4.Table S1. Crystal data and structure refinement for 1 and 2

\begin{tabular}{|c|c|c|}
\hline Identification code & 1 & 2 \\
\hline $\mathrm{CCDC}$ & 1031272 & 1031273 \\
\hline Empirical formula & C70 H48 N8 O12 Zn3 & $\mathrm{C} 70 \mathrm{H} 48 \mathrm{Cd} 3 \mathrm{~N} 8 \mathrm{O} 12$ \\
\hline Formula weight & 1389.27 & 1530.36 \\
\hline Temperature (K) & 298.15 & 259.15 \\
\hline Wavelength $(\AA)$ & 0.71073 & 0.71073 \\
\hline Crystal system & Monoclinic & Orthorhombic \\
\hline Space group & C121 & $\mathrm{P} 2(1) 2(1) 2(1)$ \\
\hline Unit cell dimensions & $\begin{array}{l}\mathrm{a}=21.719(3) \AA \\
\mathrm{b}=19.469(2) \AA \\
\mathrm{c}=20.835(2) \AA \\
\alpha=\gamma=90^{\circ}, \beta=108.856^{\circ}\end{array}$ & $\begin{array}{l}a=11.6274(18) A \\
b=19.663(4) \AA \\
c=32.678(6) \AA \\
\alpha=\beta=\gamma=90^{\circ}\end{array}$ \\
\hline Volume $\left(\AA^{3}\right), Z$ & $8337.1(17)$ & $7471(2)$ \\
\hline Density (calculated) $\left(\mathrm{mg} / \mathrm{m}^{3}\right)$ & 1.107 & 1.361 \\
\hline Absorption coefficient $\left(\mathrm{mm}^{-1}\right)$ & 0.910 & 0.906 \\
\hline $\mathrm{F}(000)$ & 2840 & 3056 \\
\hline$\theta$ range for data collection $\left(^{\circ}\right)$ & 1.575 to 27.571 & 1.620 to 27.528 \\
\hline Limiting indices & $\begin{array}{l}-28<=\mathrm{h}<=28 \\
-25<=\mathrm{k}<=25 \\
-27<=\mathrm{l}<=27\end{array}$ & $\begin{array}{l}-11<=\mathrm{h}<=14 \\
-7<=\mathrm{k}<=25 \\
-42<=\mathrm{l}<=28\end{array}$ \\
\hline Reflections collected & $107863 / 19100$ & $18498 / 14829$ \\
\hline Independent reflections & $\mathrm{R}($ int $)=0.0856$ & $\mathrm{R}($ int $)=0.0526$ \\
\hline Completeness to theta & $99.9 \%$ & $97.6 \%$ \\
\hline Refinement method & $\begin{array}{l}\text { Full-matrix least-squares on } \\
\mathrm{F}^{2}\end{array}$ & $\begin{array}{l}\text { Full-matrix least-squares on } \\
\mathrm{F}^{2}\end{array}$ \\
\hline Data / restraints / parameters & $19100 / 69 / 814$ & $14829 / 215 / 790$ \\
\hline Goodness-of-fit on $\mathrm{F}^{\wedge} 2$ & 0.953 & 0.982 \\
\hline Final R indices $[\mathrm{I}>2 \operatorname{sigma}(\mathrm{I})]$ & $\mathrm{R} 1=0.0524, \mathrm{wR} 2=0.1264$ & $\mathrm{R} 1=0.0750, \mathrm{wR} 2=0.1884$ \\
\hline $\mathrm{R}$ indices (all data) & $\mathrm{R} 1=0.1076, \mathrm{wR} 2=0.1512$ & $\mathrm{R} 1=0.1340, \mathrm{wR} 2=0.2327$ \\
\hline Absolute structure parameter & $0.052(7)$ & $0.07(3)$ \\
\hline $\begin{array}{l}\text { Largest diff. peak and hole } \\
\left(\mathrm{e} . \AA^{-3}\right)\end{array}$ & 0.477 and -0.308 & 1.500 and -0.731 \\
\hline
\end{tabular}


5. Table S2-S3. Selected bond lengths [ $[\mathbf{A}]$ and angles $\left[{ }^{0}\right]$ for 1 and 2

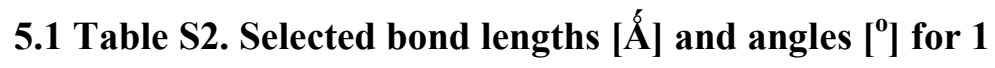

\begin{tabular}{|c|c|}
\hline $\mathrm{Zn}(1)-\mathrm{O}(11)$ & $1.962(4)$ \\
\hline $\mathrm{Zn}(1)-\mathrm{N}(2)$ & $2.009(5)$ \\
\hline $\mathrm{Zn}(1)-\mathrm{O}(1)$ & $1.936(5)$ \\
\hline $\mathrm{Zn}(1)-\mathrm{N}(8) \# 1$ & $2.072(5)$ \\
\hline $\mathrm{Zn}(2)-\mathrm{N}(7)$ & $2.007(5)$ \\
\hline $\mathrm{Zn}(2)-\mathrm{O}(3)$ & $1.973(7)$ \\
\hline $\mathrm{Zn}(2)-\mathrm{N}(1) \# 2$ & $2.044(6)$ \\
\hline $\mathrm{Zn}(2)-\mathrm{O}(9)$ & $1.944(5)$ \\
\hline $\mathrm{Zn}(3)-\mathrm{O}(5)$ & $1.939(7)$ \\
\hline $\mathrm{Zn}(3)-\mathrm{N}(4)$ & $2.049(4)$ \\
\hline $\mathrm{Zn}(3)-\mathrm{N}(5) \# 3$ & $2.01(4)$ \\
\hline $\mathrm{Zn}(3)-\mathrm{O}(7)$ & $2.020(8)$ \\
\hline$N(5)-Z n(3) \# 6$ & $2.006(3)$ \\
\hline $\mathrm{N}(8)-\mathrm{Zn}(1) \# 2$ & $2.072(5)$ \\
\hline $\mathrm{N}(1)-\mathrm{Zn}(2) \# 1$ & $2.044(6)$ \\
\hline $\mathrm{O}(11)-\mathrm{Zn}(1)-\mathrm{N}(2)$ & $95.4(2)$ \\
\hline $\mathrm{O}(11)-\mathrm{Zn}(1)-\mathrm{N}(8) \# 1$ & $112.4(2)$ \\
\hline $\mathrm{N}(2)-\mathrm{Zn}(1)-\mathrm{N}(8) \# 1$ & $108.0(2)$ \\
\hline $\mathrm{O}(1)-\mathrm{Zn}(1)-\mathrm{O}(11)$ & $104.3(2)$ \\
\hline $\mathrm{O}(1)-\mathrm{Zn}(1)-\mathrm{N}(2)$ & $124.2(2)$ \\
\hline $\mathrm{O}(1)-\mathrm{Zn}(1)-\mathrm{N}(8) \# 1$ & $111.2(2)$ \\
\hline $\mathrm{N}(7)-\mathrm{Zn}(2)-\mathrm{N}(1) \# 2$ & $109.3(2)$ \\
\hline $\mathrm{O}(3)-\mathrm{Zn}(2)-\mathrm{N}(7)$ & $124.8(3)$ \\
\hline $\mathrm{O}(3)-\mathrm{Zn}(2)-\mathrm{N}(1) \# 2$ & $109.6(3)$ \\
\hline $\mathrm{O}(9)-\mathrm{Zn}(2)-\mathrm{N}(7)$ & $94.8(2)$ \\
\hline $\mathrm{O}(9)-\mathrm{Zn}(2)-\mathrm{O}(3)$ & $106.7(3)$ \\
\hline $\mathrm{O}(9)-\mathrm{Zn}(2)-\mathrm{N}(1) \# 2$ & $110.0(3)$ \\
\hline $\mathrm{O}(5)-\mathrm{Zn}(3)-\mathrm{N}(4)$ & $108.9(3)$ \\
\hline $\mathrm{O}(5)-\mathrm{Zn}(3)-\mathrm{N}(5) \# 3$ & $111(3)$ \\
\hline $\mathrm{O}(5)-\mathrm{Zn}(3)-\mathrm{O}(7)$ & $97.2(4)$ \\
\hline $\mathrm{N}(5) \# 3-\mathrm{Zn}(3)-\mathrm{N}(4)$ & $116.0(18)$ \\
\hline $\mathrm{N}(5) \# 3-\mathrm{Zn}(3)-\mathrm{O}(7)$ & $105.8(5)$ \\
\hline $\mathrm{O}(7)-\mathrm{Zn}(3)-\mathrm{N}(4)$ & $116.1(4)$ \\
\hline $\mathrm{C}(63)-\mathrm{O}(11)-\mathrm{Zn}(1)$ & $126.8(5)$ \\
\hline $\mathrm{C}(32)-\mathrm{N}(7)-\mathrm{Zn}(2)$ & $134.5(5)$ \\
\hline $\mathrm{C}(35)-\mathrm{N}(7)-\mathrm{Zn}(2)$ & $117.9(4)$ \\
\hline $\mathrm{C}(14)-\mathrm{N}(2)-\mathrm{Zn}(1)$ & $135.6(5)$ \\
\hline $\mathrm{C}(12)-\mathrm{N}(2)-\mathrm{Zn}(1)$ & $115.0(4)$ \\
\hline $\mathrm{C}(47)-\mathrm{O}(1)-\mathrm{Zn}(1)$ & $113.9(5)$ \\
\hline
\end{tabular}




$\begin{array}{ll}\mathrm{C}(54)-\mathrm{O}(3)-\mathrm{Zn}(2) & 109.3(6) \\ \mathrm{C}(55)-\mathrm{O}(5)-\mathrm{Zn}(3) & 117.9(8) \\ \mathrm{C}(22)-\mathrm{N}(4)-\mathrm{Zn}(3) & 116.0(3) \\ \mathrm{C}(21)-\mathrm{N}(4)-\mathrm{Zn}(3) & 122.3(3) \\ \mathrm{C}(28)-\mathrm{N}(5)-\mathrm{Zn}(3) \# 6 & 116.7(3) \\ \mathrm{C}(24)-\mathrm{N}(5)-\mathrm{Zn}(3) \# 6 & 123.2(2) \\ \mathrm{C}(44)-\mathrm{N}(8)-\mathrm{Zn}(1) \# 2 & 123.1(4) \\ \mathrm{C}(45)-\mathrm{N}(8)-\mathrm{Zn}(1) \# 2 & 120.3(5) \\ \mathrm{C}(5)-\mathrm{N}(1)-\mathrm{Zn}(2) \# 1 & 121.9(5) \\ \mathrm{C}(1)-\mathrm{N}(1)-\mathrm{Zn}(2) \# 1 & 122.0(5) \\ \mathrm{C}(70)-\mathrm{O}(9)-\mathrm{Zn}(2) & 127.7(5) \\ \mathrm{C}(62)-\mathrm{O}(7)-\mathrm{Zn}(3) & 105.3(10) \\ \end{array}$

Symmetry transformations used to generate equivalent atoms:

\#1 x,y+1,z \#2 x,y-1,z \#3-x-3/2,y+1/2,-z \#4-x-3,y,-z-1

$\# 5-x-3 / 2, y+1 / 2,-z-1 \quad \# 6-x-3 / 2, y-1 / 2,-z \quad \# 7-x-3, y,-z \quad \# 8-x-3 / 2, y-1 / 2,-z-1$

5.2 Table S3.Selected bond lengths [ $[\hat{]}]$ and angles $\left[{ }^{0}\right]$ for 2

$\begin{array}{ll}\mathrm{N}(8)-\mathrm{Cd}(3) \# 1 & 2.281(8) \\ \mathrm{Cd}(1)-\mathrm{N}(5) \# 3 & 2.310(12) \\ \mathrm{Cd}(1)-\mathrm{N}(3) \# 3 & 2.276(11) \\ \mathrm{Cd}(1)-\mathrm{O}(3) & 2.325(14) \\ \mathrm{Cd}(1)-\mathrm{O}(4) & 2.460(12) \\ \mathrm{Cd}(1)-\mathrm{O}(5) & 2.387(12) \\ \mathrm{Cd}(1)-\mathrm{O}(12) & 2.387(14) \\ \mathrm{Cd}(3)-\mathrm{N}(8) \# 4 & 2.28(18) \\ \mathrm{Cd}(3)-\mathrm{C}(70) \# 5 & 2.67(2) \\ \mathrm{Cd}(3)-\mathrm{N}(1) \# 6 & 2.321(15) \\ \mathrm{Cd}(3)-\mathrm{O}(11) \# 5 & 2.275(12) \\ \mathrm{Cd}(3)-\mathrm{O}(6) \# 5 & 2.430(15) \\ \mathrm{Cd}(3)-\mathrm{O}(2) & 2.256(14) \\ \mathrm{Cd}(3)-\mathrm{O}(1) & 2.513(15) \\ \mathrm{Cd}(2)-\mathrm{N}(6) & 2.241(14) \\ \mathrm{Cd}(2)-\mathrm{N}(4) & 2.344(13) \\ \mathrm{Cd}(2)-\mathrm{O}(9) & 2.428(18) \\ \mathrm{Cd}(2)-\mathrm{O}(7) & 2.343(14) \\ \mathrm{Cd}(2)-\mathrm{O}(8) & 2.314(12) \\ \mathrm{Cd}(2)-\mathrm{O}(10) & 2.220(15) \\ \mathrm{N}(5)-\mathrm{Cd}(1) \# 7 & 2.310(12) \\ \mathrm{N}(3)-\mathrm{Cd}(1) \# 7 & 2.276(11) \\ \mathrm{N}(1)-\mathrm{Cd}(3) \# 8 & 2.321(15) \\ \mathrm{O}(11)-\mathrm{Cd}(3) \# 2 & 2.275(12) \\ & \end{array}$




\begin{tabular}{|c|c|}
\hline $\mathrm{O}(6)-\mathrm{Cd}(3) \# 2$ & $2.430(15)$ \\
\hline $\mathrm{C}(25)-\mathrm{N}(8)-\mathrm{Cd}(3) \# 1$ & $119.3(5)$ \\
\hline $\mathrm{N}(5) \# 3-\mathrm{Cd}(1)-\mathrm{O}(3)$ & $89.0(5)$ \\
\hline $\mathrm{N}(5) \# 3-\mathrm{Cd}(1)-\mathrm{O}(4)$ & $138.1(5)$ \\
\hline $\mathrm{N}(5) \# 3-\mathrm{Cd}(1)-\mathrm{O}(5)$ & $128.0(5)$ \\
\hline $\mathrm{N}(5) \# 3-\mathrm{Cd}(1)-\mathrm{O}(12)$ & $80.3(5)$ \\
\hline $\mathrm{N}(3) \# 3-\mathrm{Cd}(1)-\mathrm{C}(47)$ & $98.7(5)$ \\
\hline $\mathrm{N}(3) \# 3-\mathrm{Cd}(1)-\mathrm{N}(5) \# 3$ & $105.1(5)$ \\
\hline $\mathrm{N}(3) \# 3-\mathrm{Cd}(1)-\mathrm{O}(3)$ & $113.5(4)$ \\
\hline $\mathrm{N}(3) \# 3-\mathrm{Cd}(1)-\mathrm{O}(4)$ & $83.6(5)$ \\
\hline $\mathrm{N}(3) \# 3-\mathrm{Cd}(1)-\mathrm{O}(5)$ & $102.6(5)$ \\
\hline $\mathrm{N}(3) \# 3-\mathrm{Cd}(1)-\mathrm{O}(12)$ & $119.7(6)$ \\
\hline $\mathrm{O}(3)-\mathrm{Cd}(1)-\mathrm{O}(4)$ & $51.2(4)$ \\
\hline $\mathrm{O}(3)-\mathrm{Cd}(1)-\mathrm{O}(5)$ & $118.3(6)$ \\
\hline $\mathrm{O}(3)-\mathrm{Cd}(1)-\mathrm{O}(12)$ & $126.7(6)$ \\
\hline $\mathrm{O}(5)-\mathrm{Cd}(1)-\mathrm{O}(4)$ & $87.7(5)$ \\
\hline $\mathrm{O}(12)-\mathrm{Cd}(1)-\mathrm{O}(4)$ & $131.2(5)$ \\
\hline $\mathrm{O}(12)-\mathrm{Cd}(1)-\mathrm{O}(5)$ & $47.7(4)$ \\
\hline $\mathrm{N}(8) \# 4-\mathrm{Cd}(3)-\mathrm{N}(1) \# 6$ & $95(5)$ \\
\hline $\mathrm{N}(8) \# 4-\mathrm{Cd}(3)-\mathrm{O}(6) \# 5$ & $95(4)$ \\
\hline N(8)\#4-Cd(3)-O(1) & $105(3)$ \\
\hline $\mathrm{N}(1) \# 6-\mathrm{Cd}(3)-\mathrm{O}(6) \# 5$ & $122.8(5)$ \\
\hline $\mathrm{N}(1) \# 6-\mathrm{Cd}(3)-\mathrm{O}(1)$ & $87.3(5)$ \\
\hline $\mathrm{O}(11) \# 5-\mathrm{Cd}(3)-\mathrm{N}(8) \# 4$ & $143(3)$ \\
\hline $\mathrm{O}(11) \# 5-\mathrm{Cd}(3)-\mathrm{N}(1) \# 6$ & $89.3(5)$ \\
\hline $\mathrm{O}(11) \# 5-\mathrm{Cd}(3)-\mathrm{O}(6) \# 5$ & $53.8(4)$ \\
\hline $\mathrm{O}(11) \# 5-\mathrm{Cd}(3)-\mathrm{O}(1)$ & $111.8(7)$ \\
\hline $\mathrm{O}(6) \# 5-\mathrm{Cd}(3)-\mathrm{O}(1)$ & $142.8(6)$ \\
\hline $\mathrm{O}(2)-\mathrm{Cd}(3)-\mathrm{N}(8) \# 4$ & $102(4)$ \\
\hline $\mathrm{O}(2)-\mathrm{Cd}(3)-\mathrm{N}(1) \# 6$ & $138.3(7)$ \\
\hline $\mathrm{O}(2)-\mathrm{Cd}(3)-\mathrm{O}(11) \# 5$ & 98.1(9) \\
\hline $\mathrm{O}(2)-\mathrm{Cd}(3)-\mathrm{O}(6) \# 5$ & $93.5(5)$ \\
\hline $\mathrm{O}(2)-\mathrm{Cd}(3)-\mathrm{O}(1)$ & $51.8(5)$ \\
\hline $\mathrm{N}(6)-\mathrm{Cd}(2)-\mathrm{N}(4)$ & $96.9(5)$ \\
\hline $\mathrm{N}(6)-\mathrm{Cd}(2)-\mathrm{O}(9)$ & $119.1(6)$ \\
\hline $\mathrm{N}(6)-\mathrm{Cd}(2)-\mathrm{O}(7)$ & $86.8(5)$ \\
\hline $\mathrm{N}(6)-\mathrm{Cd}(2)-\mathrm{O}(8)$ & $130.5(5)$ \\
\hline $\mathrm{N}(4)-\mathrm{Cd}(2)-\mathrm{O}(9)$ & $129.5(6)$ \\
\hline $\mathrm{O}(7)-\mathrm{Cd}(2)-\mathrm{N}(4)$ & $121.3(5)$ \\
\hline $\mathrm{O}(7)-\mathrm{Cd}(2)-\mathrm{O}(9)$ & $96.5(6)$ \\
\hline $\mathrm{O}(8)-\mathrm{Cd}(2)-\mathrm{N}(4)$ & $85.5(4)$ \\
\hline $\mathrm{O}(8)-\mathrm{Cd}(2)-\mathrm{O}(9)$ & $94.8(7)$ \\
\hline $\mathrm{O}(8)-\mathrm{Cd}(2)-\mathrm{O}(7)$ & $52.2(5)$ \\
\hline
\end{tabular}




$\begin{array}{ll}\mathrm{O}(10)-\mathrm{Cd}(2)-\mathrm{N}(6) & 110.2(7) \\ \mathrm{O}(10)-\mathrm{Cd}(2)-\mathrm{N}(4) & 84.2(7) \\ \mathrm{O}(10)-\mathrm{Cd}(2)-\mathrm{O}(9) & 51.8(6) \\ \mathrm{O}(10)-\mathrm{Cd}(2)-\mathrm{O}(7) & 148.2(7) \\ \mathrm{O}(10)-\mathrm{Cd}(2)-\mathrm{O}(8) & 119.3(7) \\ \mathrm{C}(44)-\mathrm{N}(5)-\mathrm{Cd}(1) \# 7 & 124.6(13) \\ \mathrm{C}(45)-\mathrm{N}(5)-\mathrm{Cd}(1) \# 7 & 120.3(11) \\ \mathrm{C}(9)-\mathrm{N}(3)-\mathrm{Cd}(1) \# 7 & 128.7(9) \\ \mathrm{C}(12)-\mathrm{N}(3)-\mathrm{Cd}(1) \# 7 & 123.6(9) \\ \mathrm{C}(31)-\mathrm{N}(6)-\mathrm{Cd}(2) & 126.9(10) \\ \mathrm{C}(35)-\mathrm{N}(6)-\mathrm{Cd}(2) & 124.9(10) \\ \mathrm{C}(22)-\mathrm{N}(4)-\mathrm{Cd}(2) & 124.1(10) \\ \mathrm{C}(21)-\mathrm{N}(4)-\mathrm{Cd}(2) & 116.2(11) \\ \mathrm{C}(5)-\mathrm{N}(1)-\mathrm{Cd}(3) \# 8 & 126.6(12) \\ \mathrm{C}(1)-\mathrm{N}(1)-\mathrm{Cd}(3) \# 8 & 112.3(14) \\ \mathrm{C}(70)-\mathrm{O}(11)-\mathrm{Cd}(3) \# 2 & 94.7(11) \\ \mathrm{C}(70)-\mathrm{O}(6)-\mathrm{Cd}(3) \# 2 & 88.3(14) \\ \mathrm{C}(47)-\mathrm{O}(3)-\mathrm{Cd}(1) & 99.2(12) \\ \mathrm{C}(63)-\mathrm{O}(9)-\mathrm{Cd}(2) & 88.7(15) \\ \mathrm{C}(62)-\mathrm{O}(7)-\mathrm{Cd}(2) & 99.2(8) \\ \mathrm{C}(62)-\mathrm{O}(8)-\mathrm{Cd}(2) & 104.9(9) \\ \mathrm{C}(63)-\mathrm{O}(10)-\mathrm{Cd}(2) & 97.5(15) \\ \mathrm{C}(47)-\mathrm{O}(4)-\mathrm{Cd}(1) & 87.9(9) \\ \mathrm{C}(54)-\mathrm{O}(2)-\mathrm{Cd}(3) & 100.9(14) \\ \mathrm{C}(54)-\mathrm{O}(1)-\mathrm{Cd}(3) & 86.4(13) \\ \mathrm{C}(55)-\mathrm{O}(5)-\mathrm{Cd}(1) & 102.1(9) \\ \mathrm{C}(55)-\mathrm{O}(12)-\mathrm{Cd}(1) & 101.3(9)\end{array}$

Symmetry transformations used to generate equivalent atoms:

$\# 1 \mathrm{x}-3 / 2,-\mathrm{y}+5 / 2,-\mathrm{z}+1 \quad \# 2-\mathrm{x}+1 / 2,-\mathrm{y}+3, \mathrm{z}-1 / 2 \quad \# 3-\mathrm{x}+2, \mathrm{y}+1 / 2,-\mathrm{z}+1 / 2 \quad \# 4 \mathrm{x}+3 / 2,-\mathrm{y}+5 / 2,-\mathrm{z}+1$

$\# 5-x+1 / 2,-y+3, z+1 / 2 \quad \# 6-x+2, y+3 / 2,-z+1 / 2 \quad \# 7-x+2, y-1 / 2,-z+1 / 2 \quad \# 8-x+2, y-3 / 2,-z+1 / 2$ 
6. Figures S1-S3. Additional X-ray crystallographic structures

6.1 Figure S1. The coordination environments of $\mathrm{Zn}$ ions in MOF 1

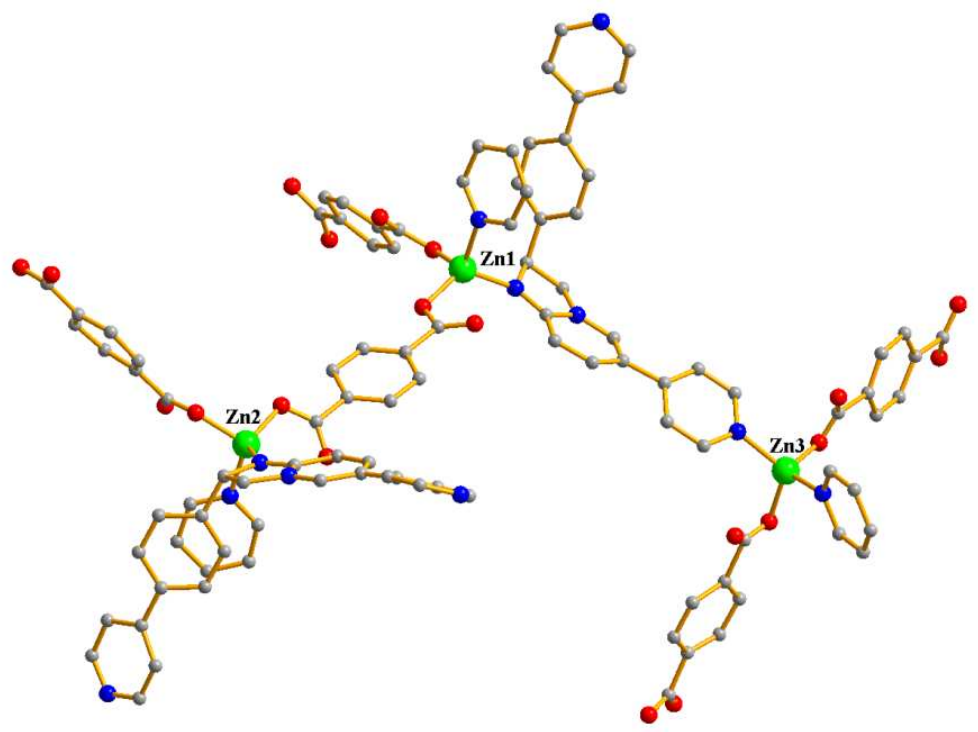

6.2 Figure S2. View of $2_{1}$ helical polymeric chain in 1

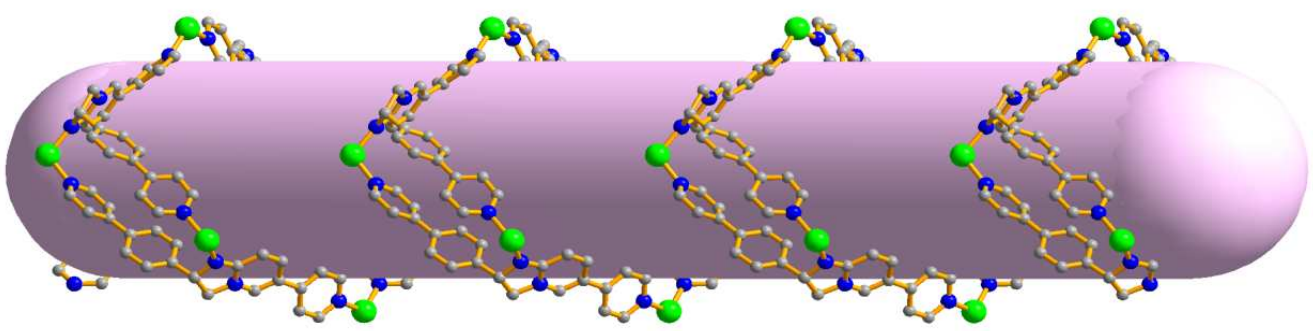

6.3 Figure S3. A view of 3D interpenetrated framework of 1 along the $c$-axis

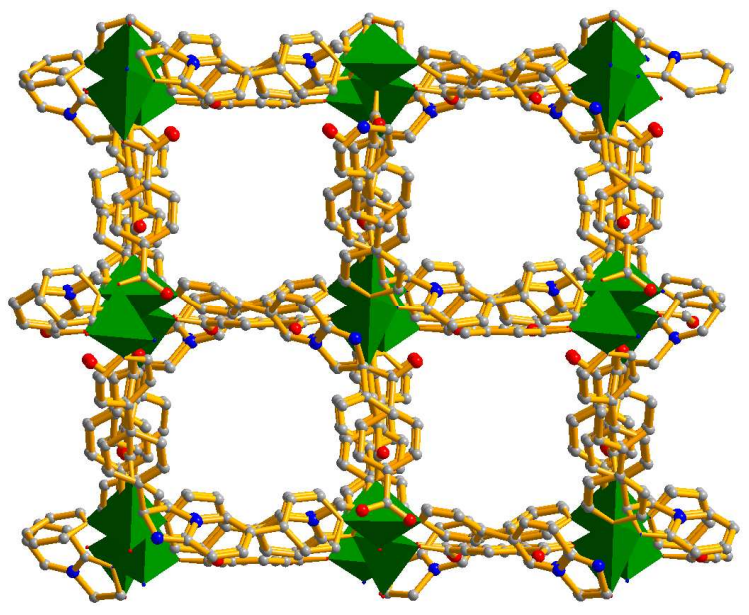


6.4 Figure S4. Scheme showing the independent network of 1

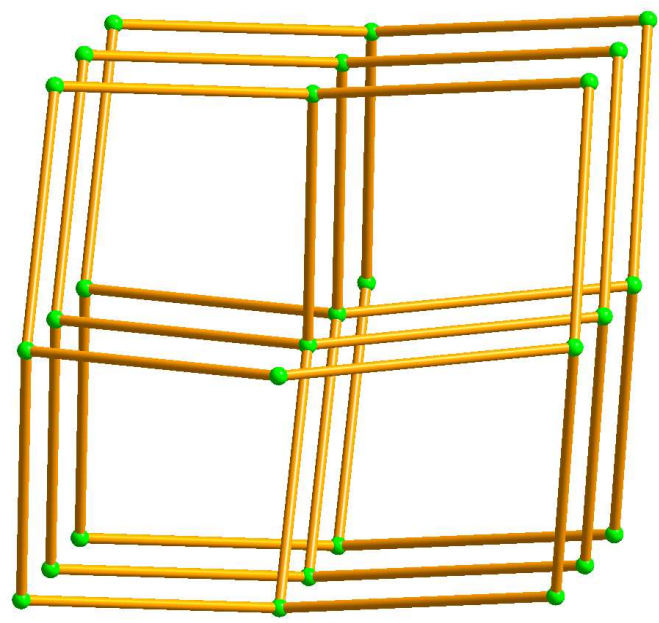

6.5 Figure S5. The coordination environments of Cd ions in MOF 2

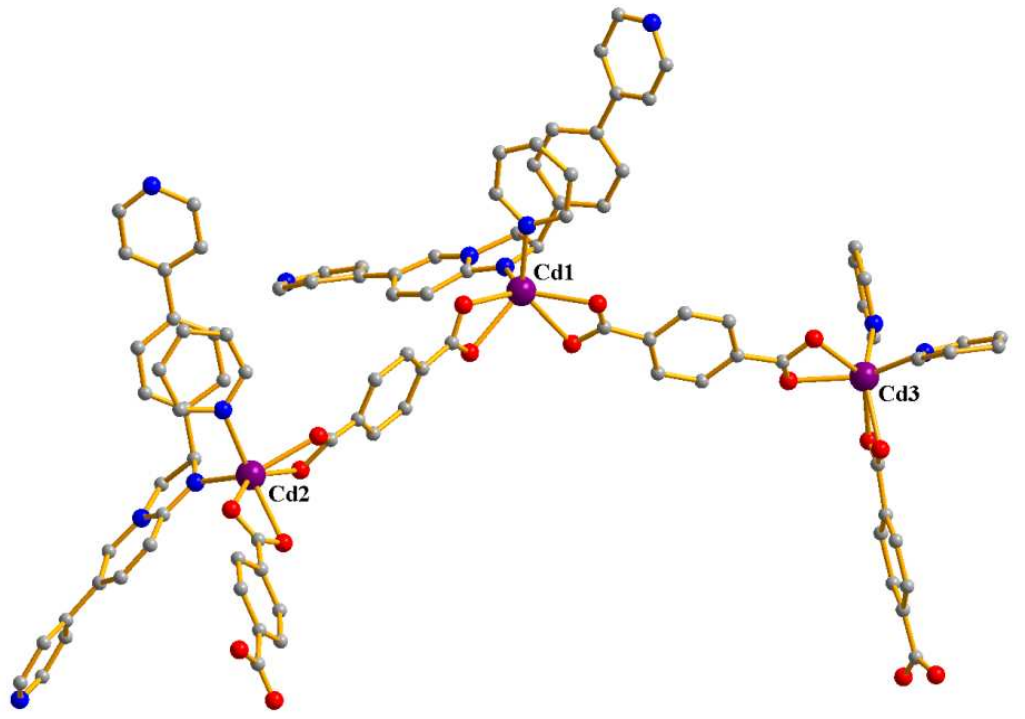

6.6 Figure S6. View of $2_{1}$ helical polymeric chain in 2

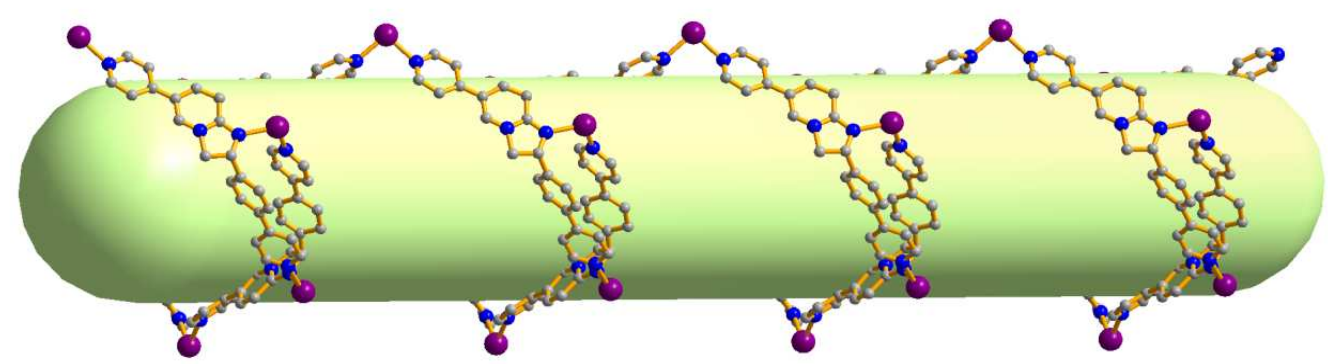


6.7 Figure S7. View of one independent network of 2 along the $c$ axis (a) and Space-filling model of 3D interpenetrated framework of 2 along the $a$-axis (b).

(a)

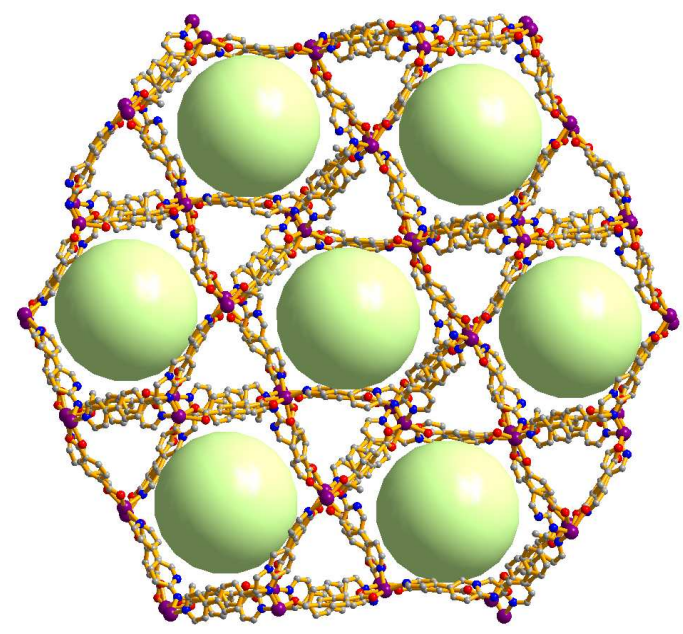

(b)

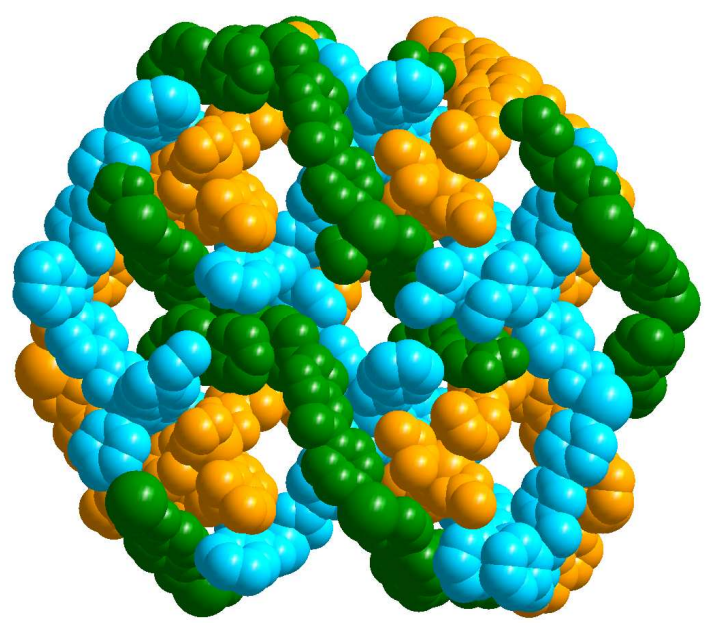

6.8 Figure S8. Scheme showing the independent network of 2

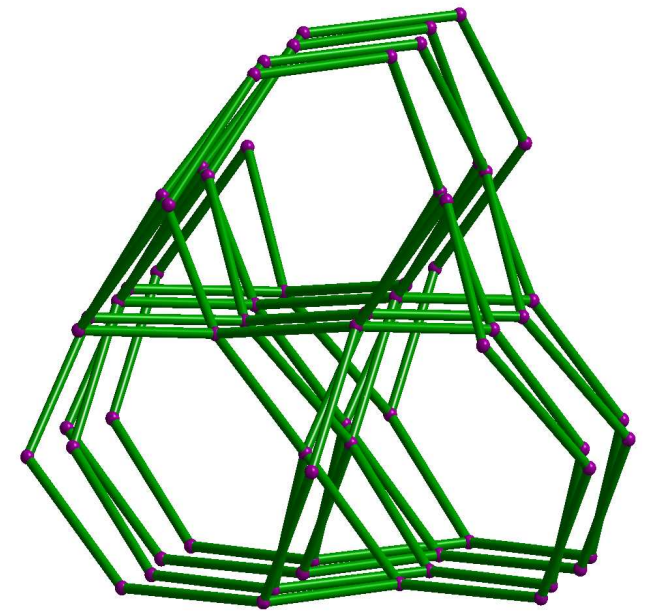




\section{Figure S9. ESI-MS spectra}

(a)

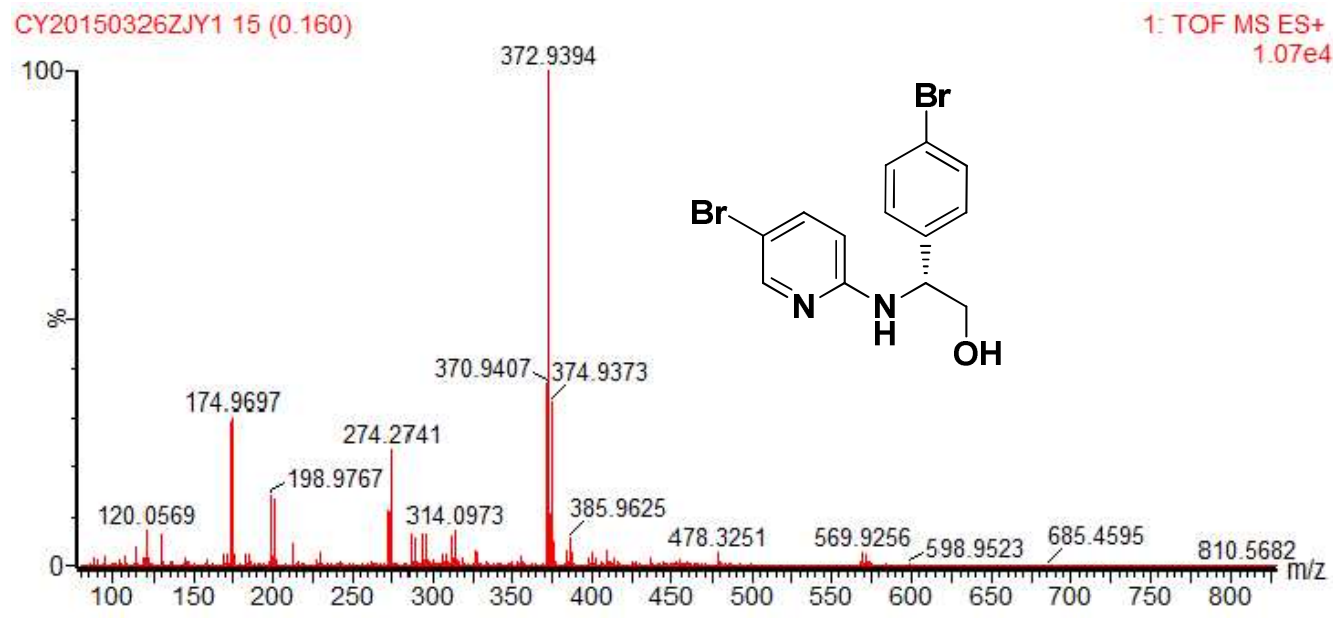

\section{(b)}

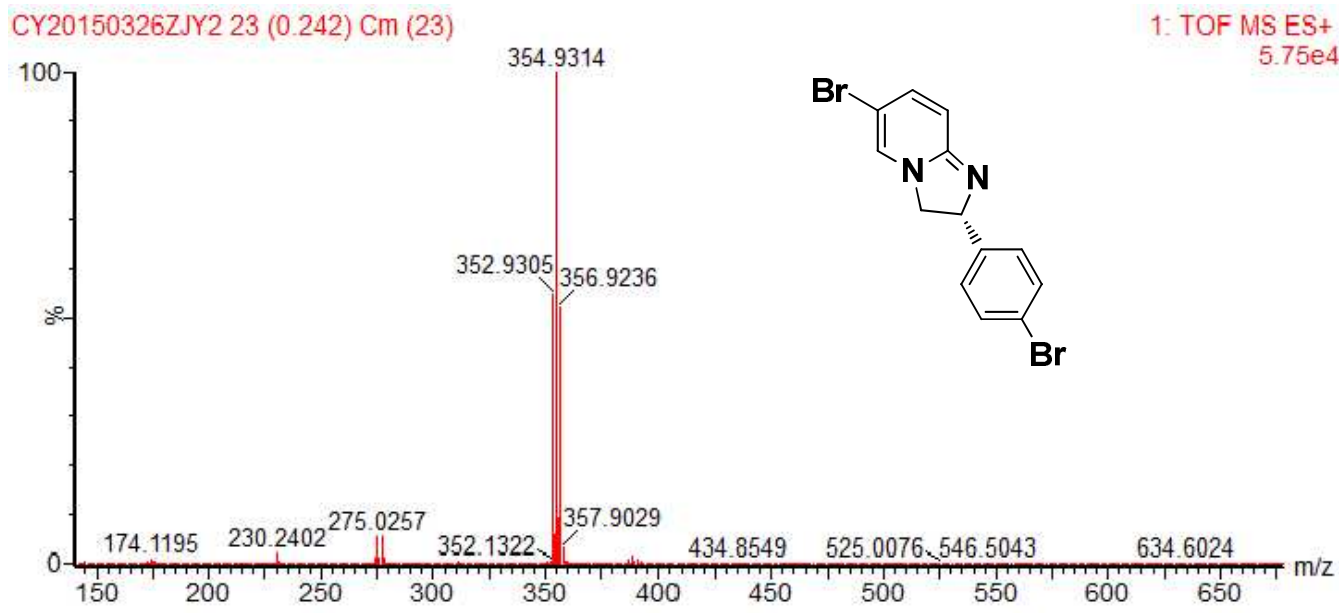

(c)

CY20150326ZJY3 13 (0.139) Cm (13:14)

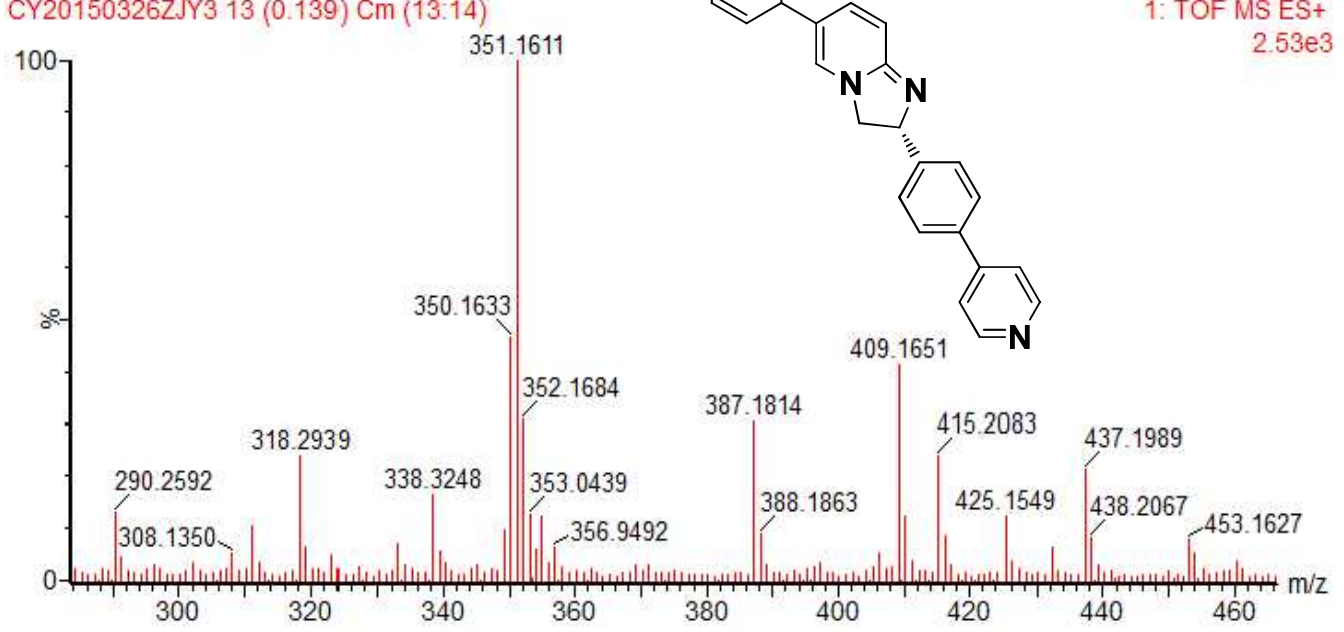




\section{Figure S10. PXRD patterns}

(a)

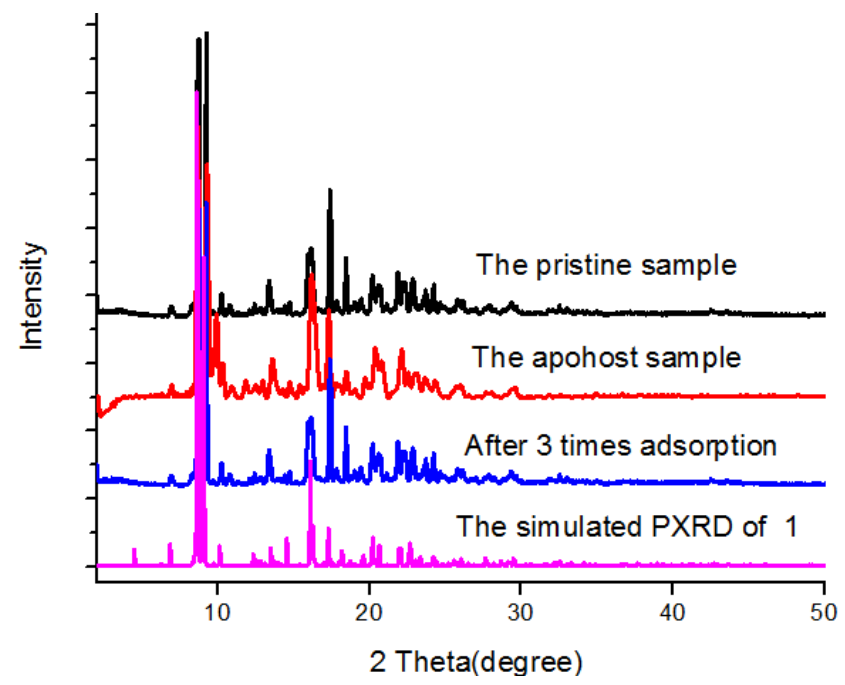

(b)

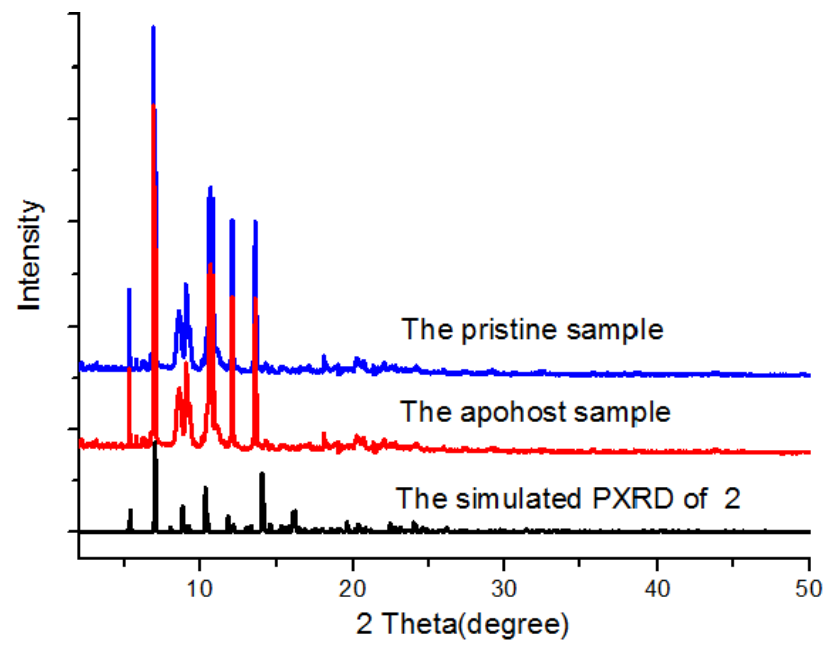

\section{Figure S11. TGA curves}

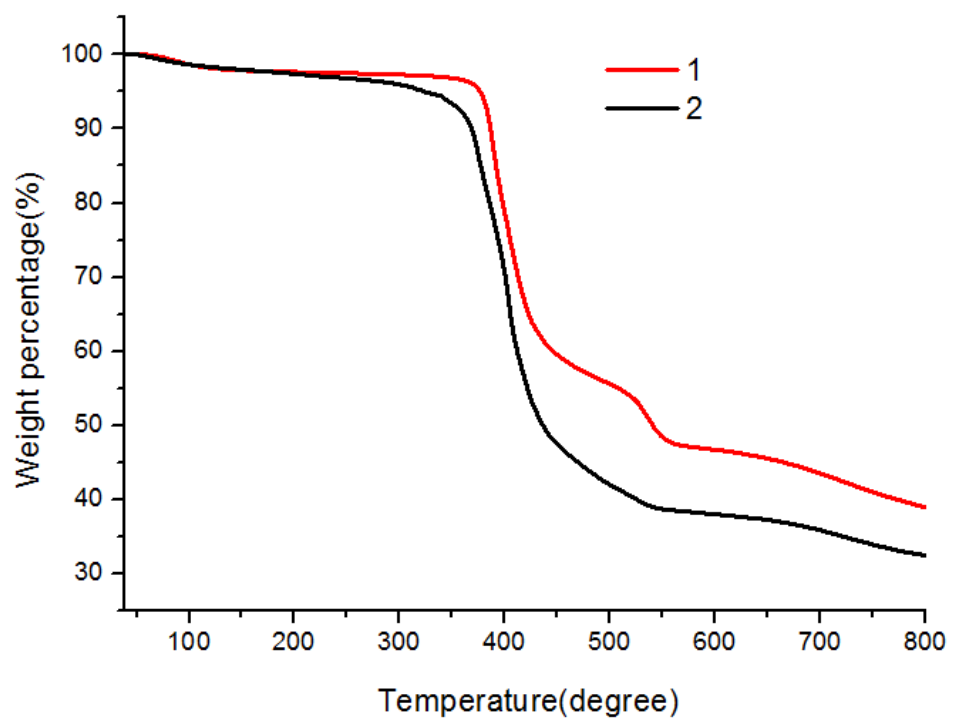


10. Figure S12. Solid-state CD spectra

(a)

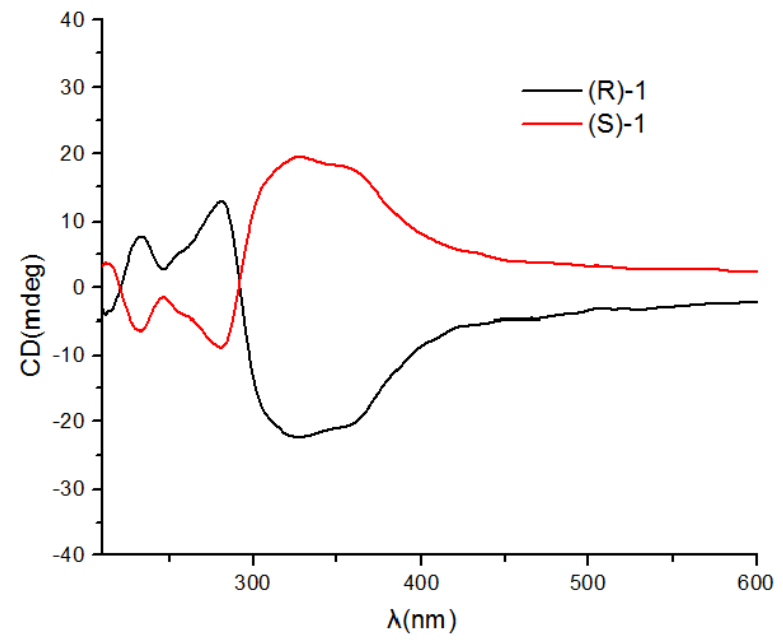

(b)

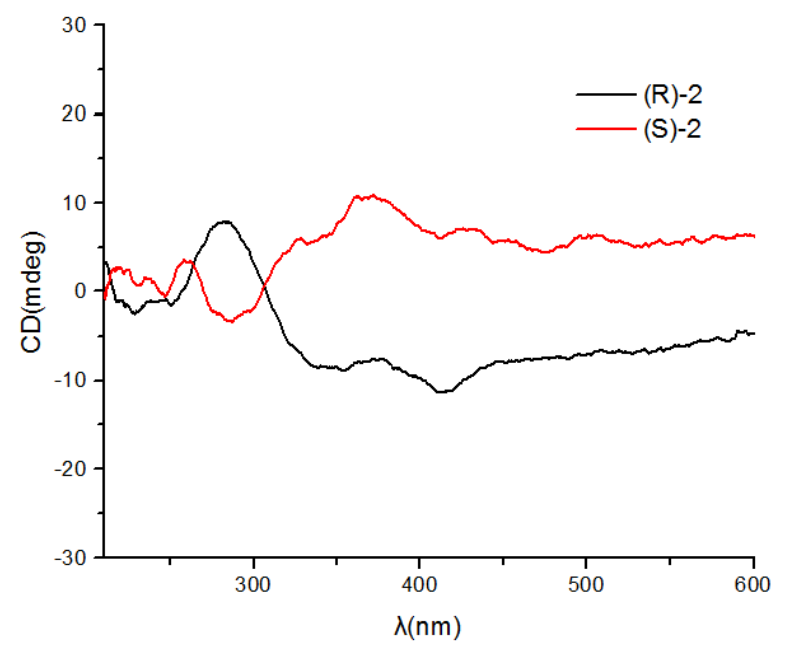

11. Figure S13. The $\mathrm{CO}_{2}$ adsorption isotherms

(a)

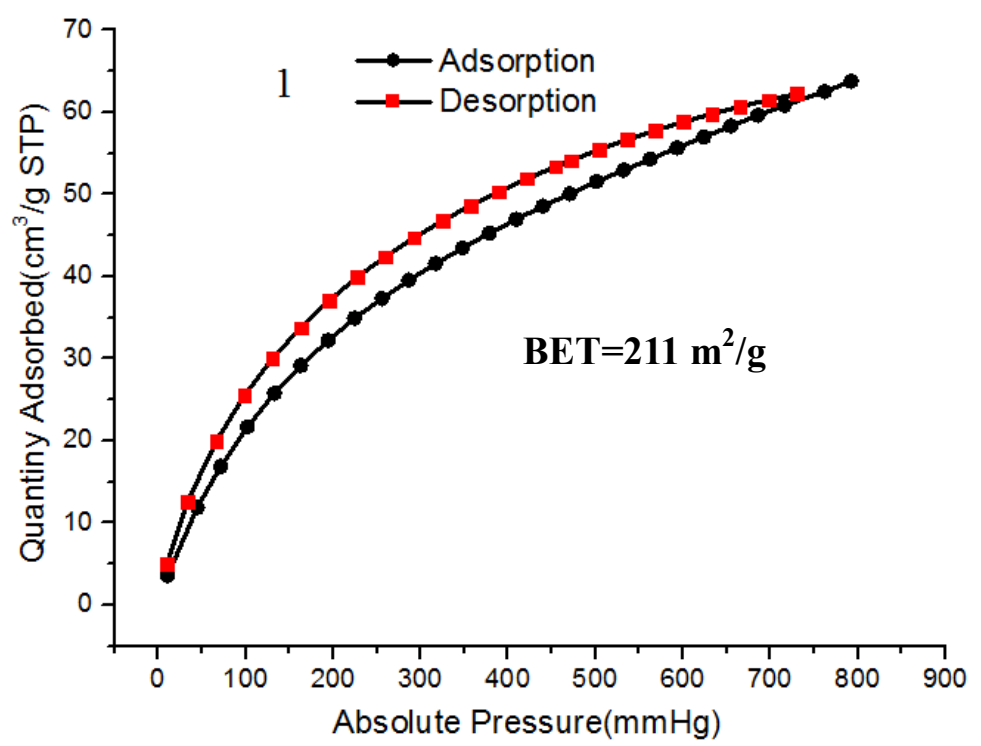


(b)

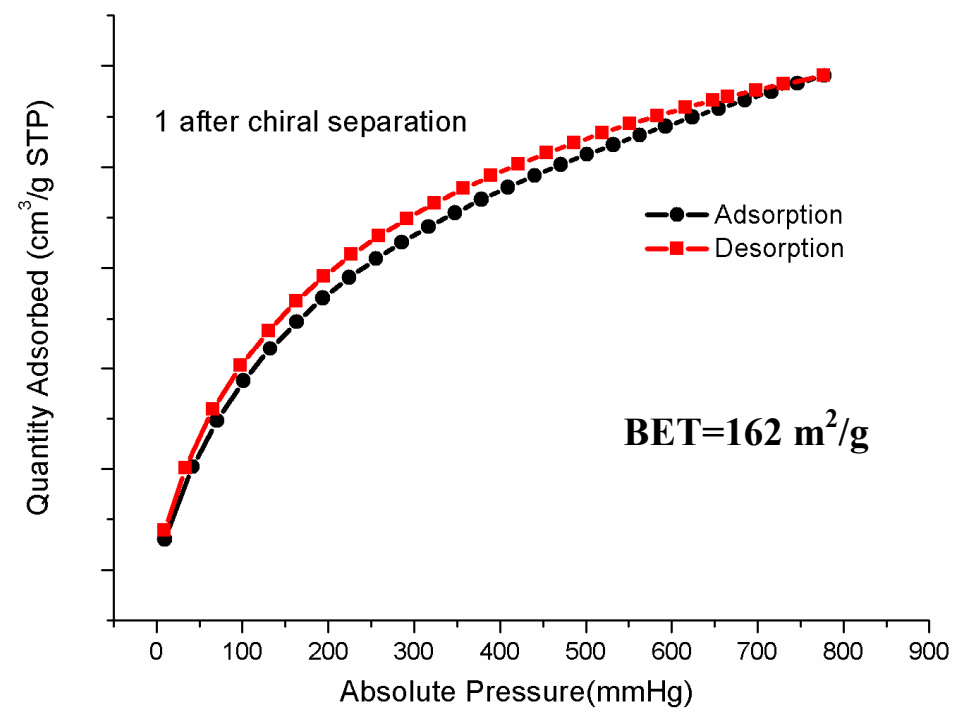

(c)

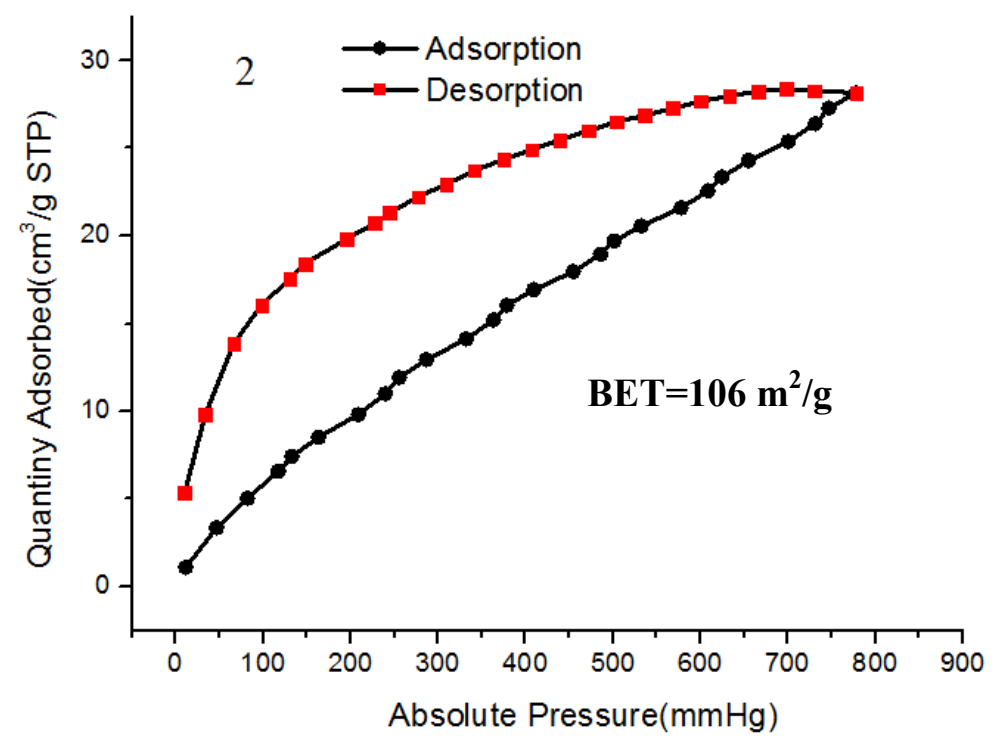

The theoretical surface areas of MOFs $\mathbf{1}$ and $\mathbf{2}$ were calculated to be 256.5 and $235.8 \mathrm{~m}^{2} / \mathrm{g}$, respectively, using Düren code. (T. Düren, F. Millange, G. Férey, K. $\mathbf{~ S}$. Walton, R. Q. Snurr, J. Phys. Chem. C., 2007, 111, 15350-15356.) For MOF 1, the observed surface area is almost comparable with the calculated one. But the surface area of $\mathbf{2}$ is obviously smaller probably due to the partial framework collapse upon removal of guest solvent molecules.

The apparent adsorption-desorption hysteresis was probably as a consequence of the framework flexibility. 
12. NMR spectra

(a) ${ }^{1} \mathrm{H}$ NMR and ${ }^{13} \mathrm{C}$ NMR of a

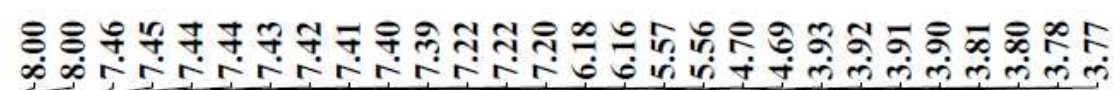

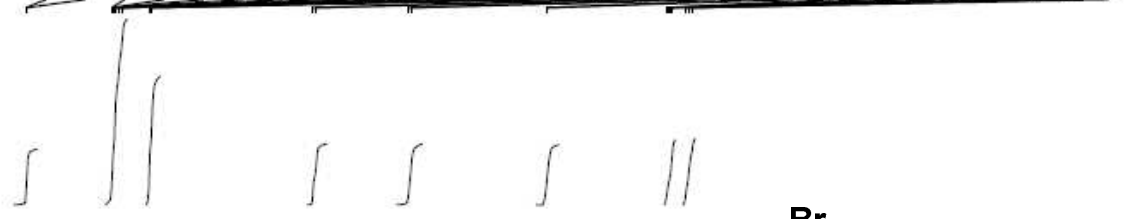<smiles>OC[C@H](Nc1ccc(Br)cn1)c1ccc(Br)cc1</smiles>

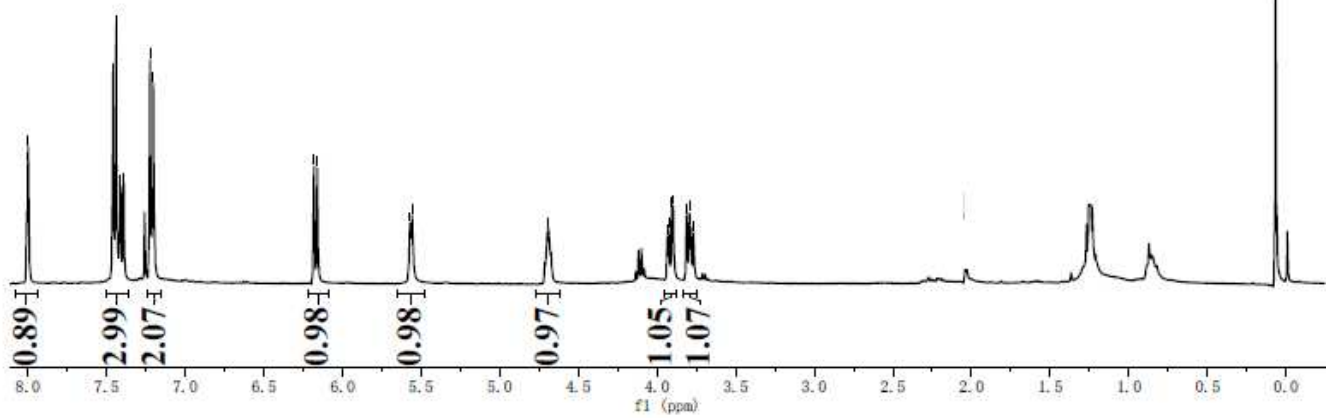

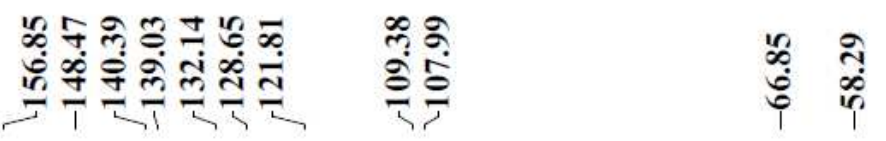
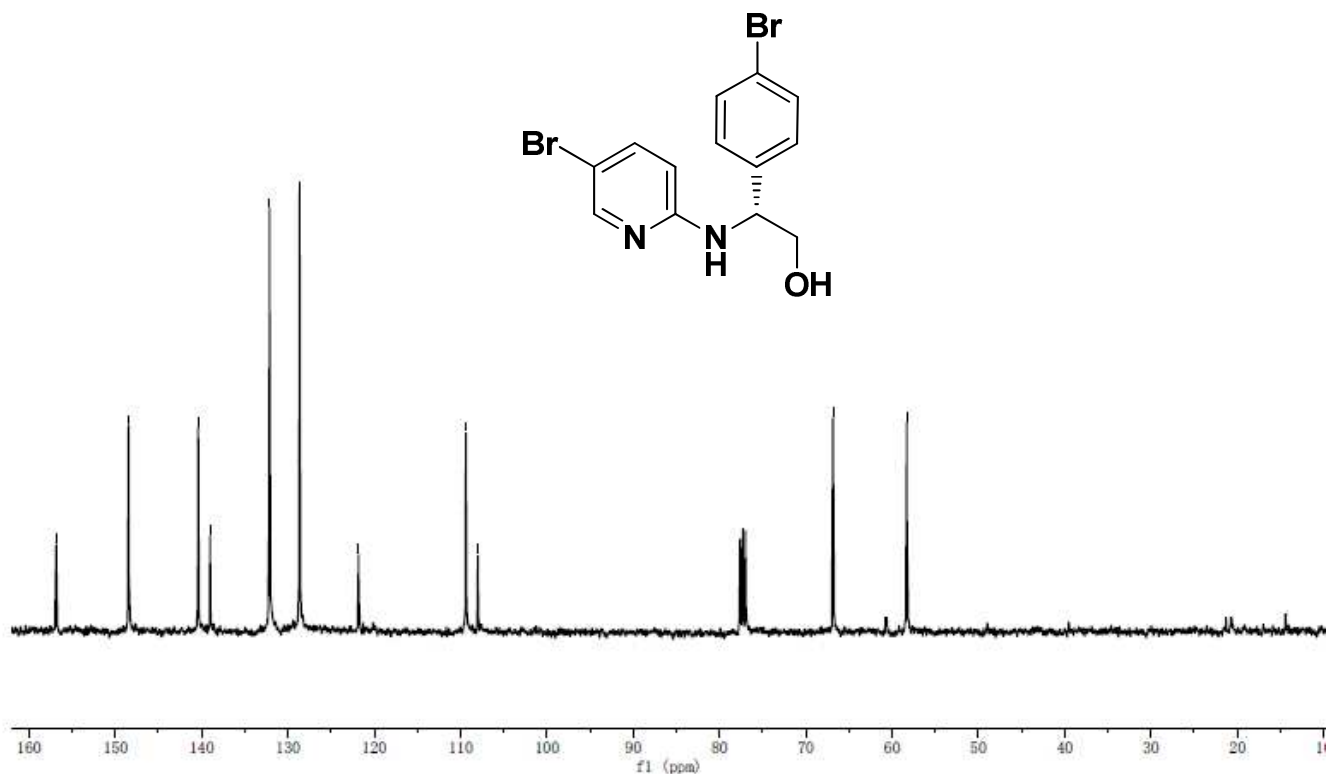


\section{(b) ${ }^{1} \mathrm{H}$ NMR and ${ }^{13} \mathrm{C}$ NMR of $b$}
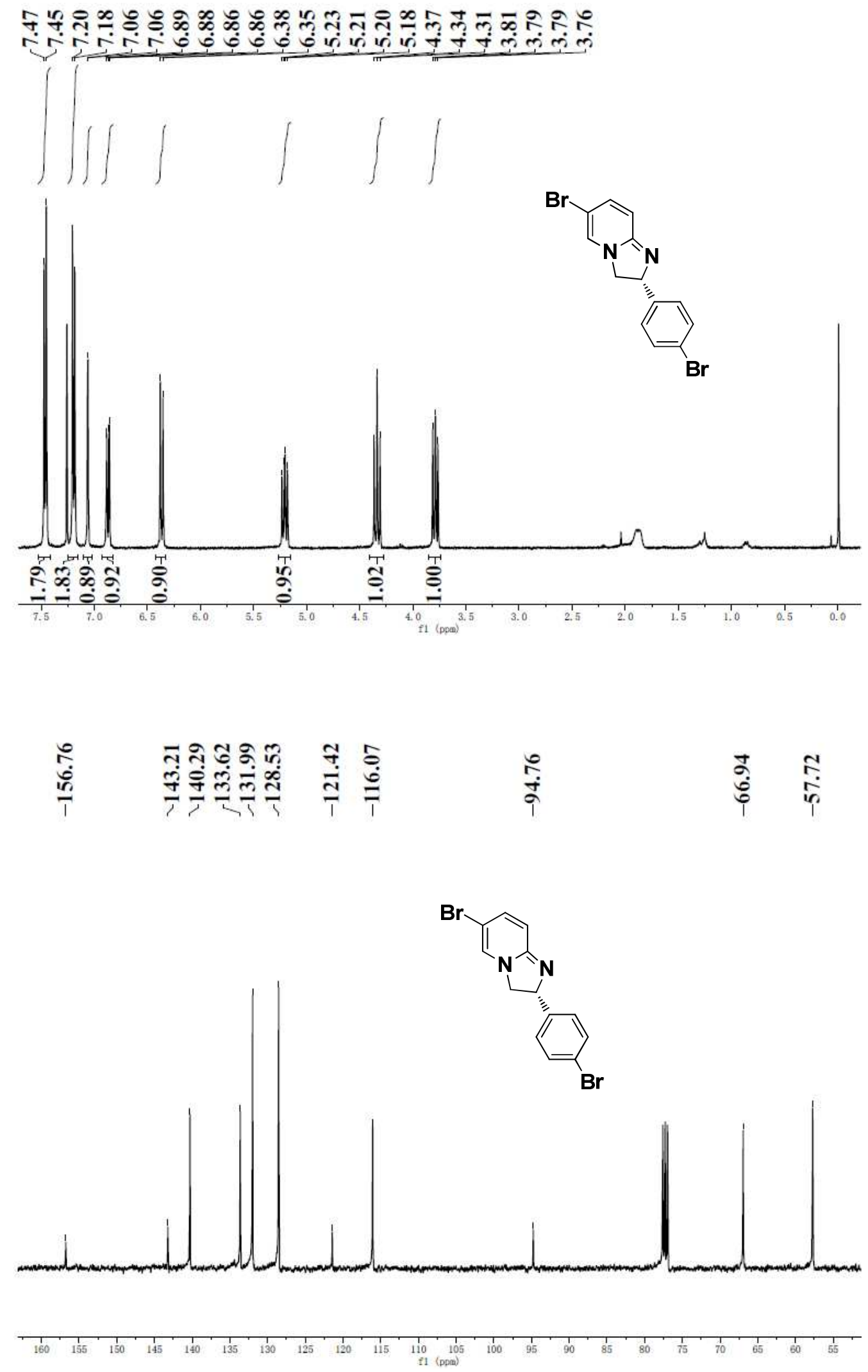
(c) ${ }^{1}$ H NMR and ${ }^{13} C$ NMR of $L$

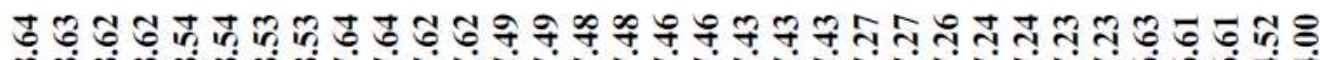

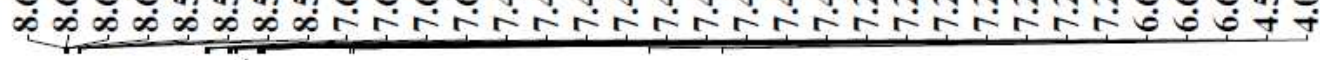
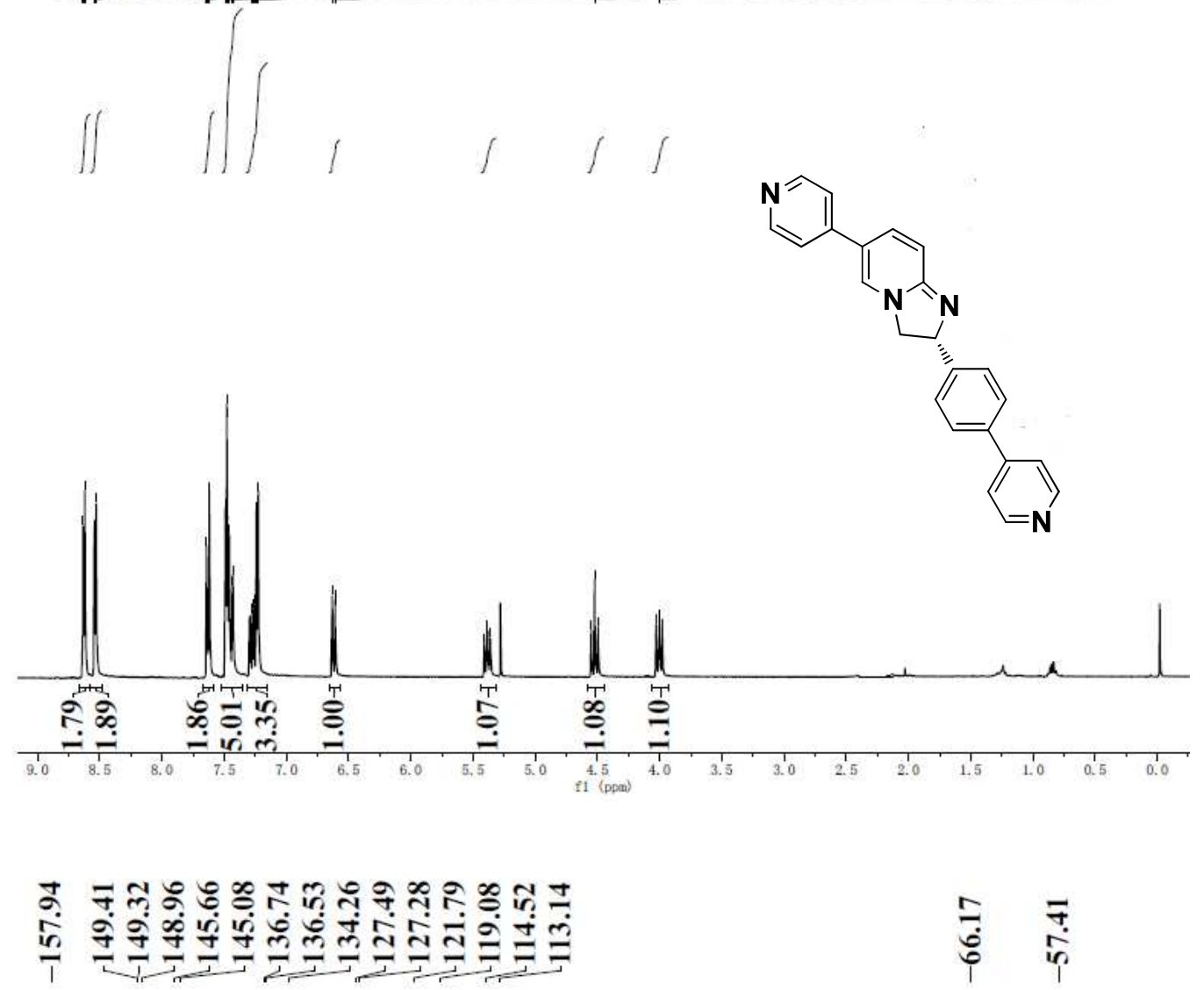

홍
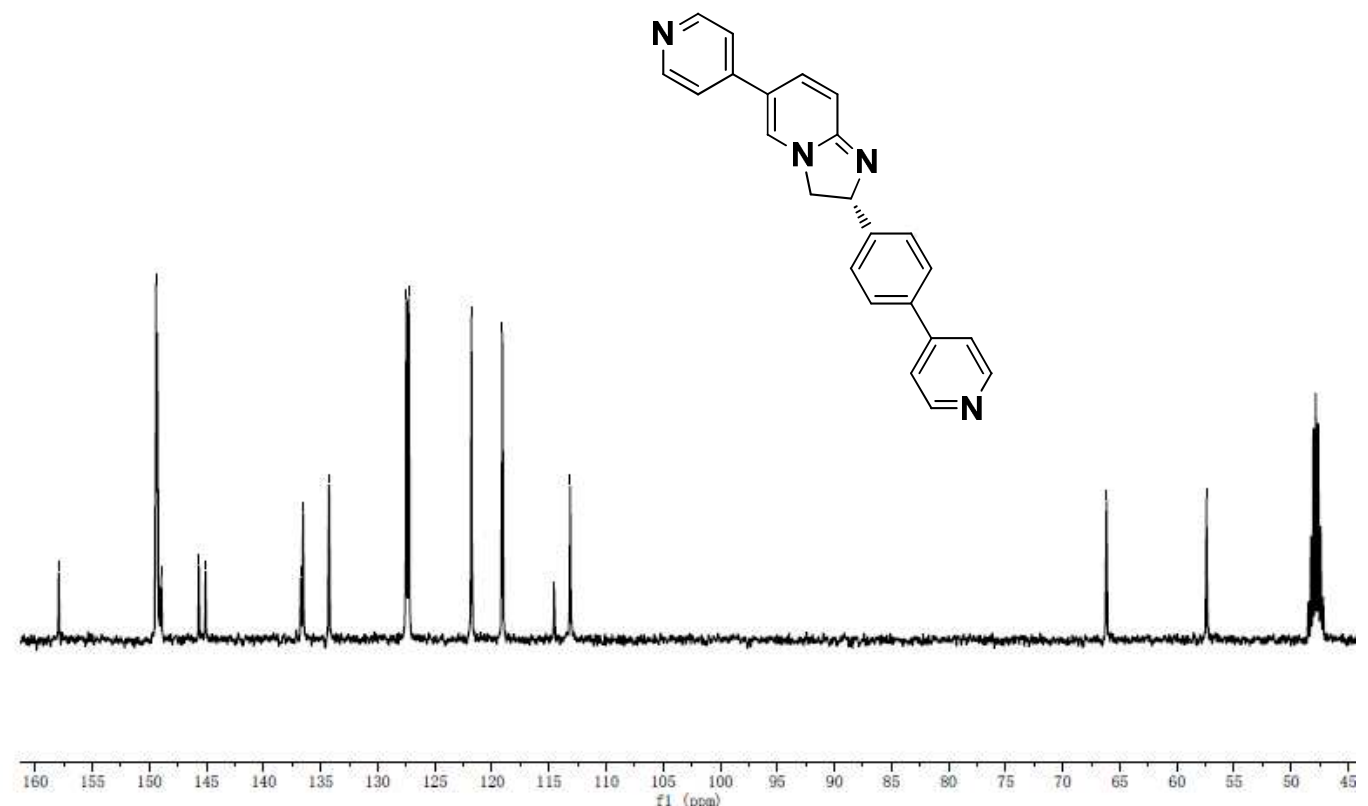


\section{HPLC results}

\subsection{HPLC of adsorption separation}<smiles>CS(=O)c1ccccc1</smiles>

Optical purity is determined by HPLC with a chiralcel OD-H column (hexane/i-PrOH $=93 / 7,1.0 \mathrm{~mL} / \mathrm{min})$, tminor $=15.74 \mathrm{~min}$, tmajor $=23.50 \mathrm{~min}$; ee $=46.8 \%$.
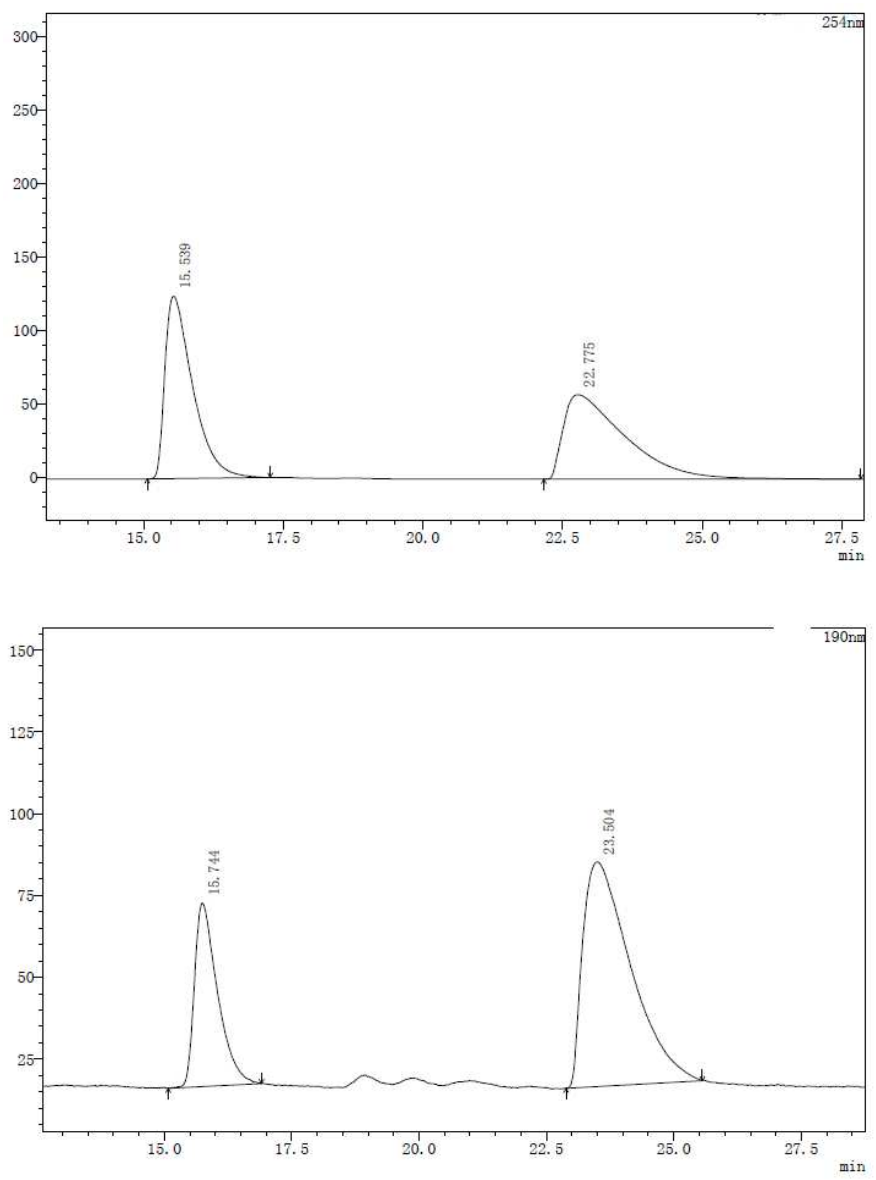

\begin{tabular}{|c|c|c|c|c|}
\hline Serial number & $\begin{array}{c}\text { Retention } \\
\text { Time }[\mathrm{min}]\end{array}$ & Area[mAbs*s] & Type & Area\% \\
\hline 1 & 15.744 & 1684895 & FF & 26.607 \\
\hline 2 & 23.504 & 4647733 & FF & 73.393 \\
\hline The Total & & 6332628 & & \\
\hline
\end{tabular}


<smiles>CCS(=O)c1ccccc1</smiles>

Optical purity is determined by HPLC with a chiralcel OD-H column (hexane/i-PrOH $=93 / 7,1.0 \mathrm{~mL} / \mathrm{min}), t_{\text {major }}=12.58 \mathrm{~min}, \mathrm{t}_{\text {minor }}=17.50 \mathrm{~min}$; ee $=20.92 \%$.
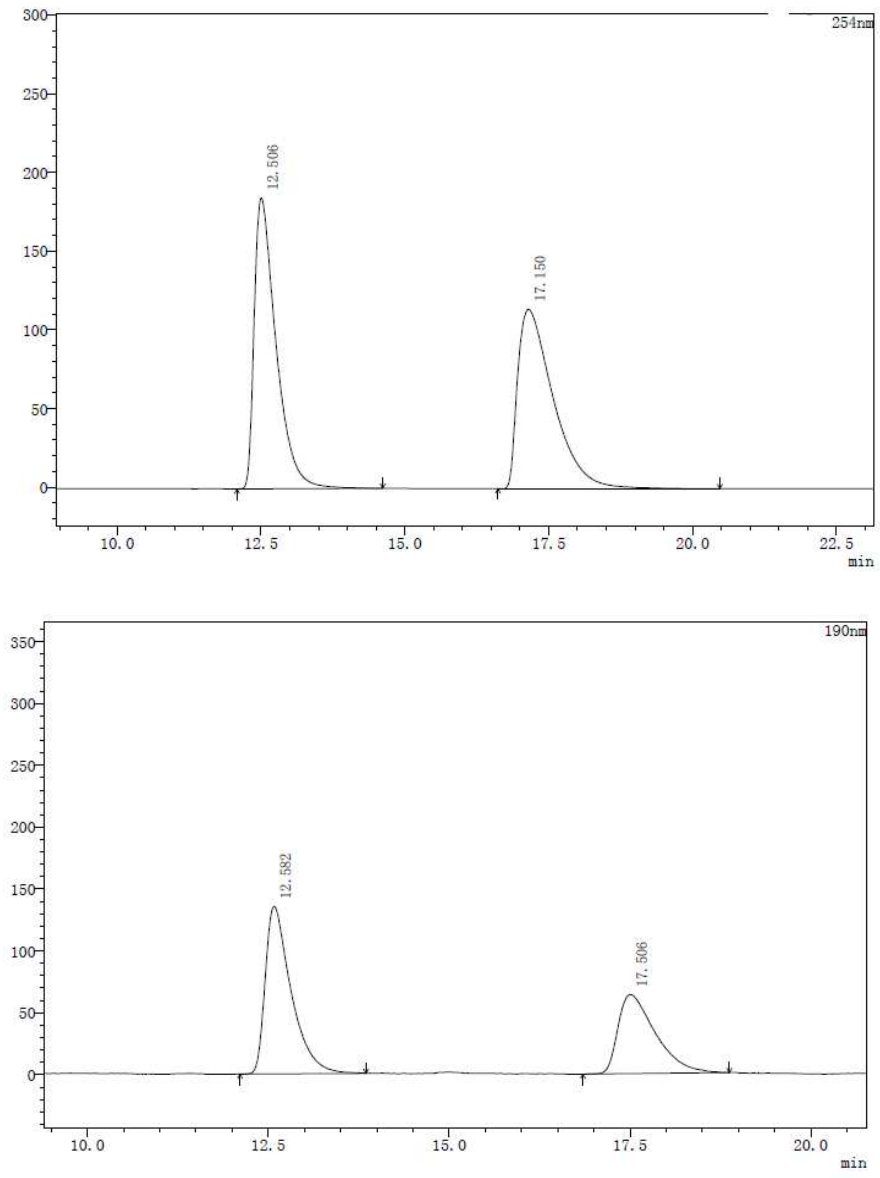

\begin{tabular}{|c|c|c|c|c|}
\hline Serial number & $\begin{array}{c}\text { Retention } \\
\text { Time }[\mathrm{min}]\end{array}$ & Area[mAbs*s] & Type & Area\% \\
\hline 1 & 12.582 & 3367495 & FF & 60.462 \\
\hline 2 & 17.506 & 2202085 & FF & 39.538 \\
\hline The Total & & 5569579 & & \\
\hline
\end{tabular}


<smiles>Cc1ccc(S(C)=O)cc1</smiles>

Optical purity is determined by HPLC with a chiralcel OD-H column (hexane/i-PrOH $=90 / 10,1.0 \mathrm{~mL} / \mathrm{min}), \mathrm{t}_{\text {minor }}=10.58 \mathrm{~min}, \mathrm{t}_{\text {major }}=11.70 \mathrm{~min}$; ee $=34.8 \%$.
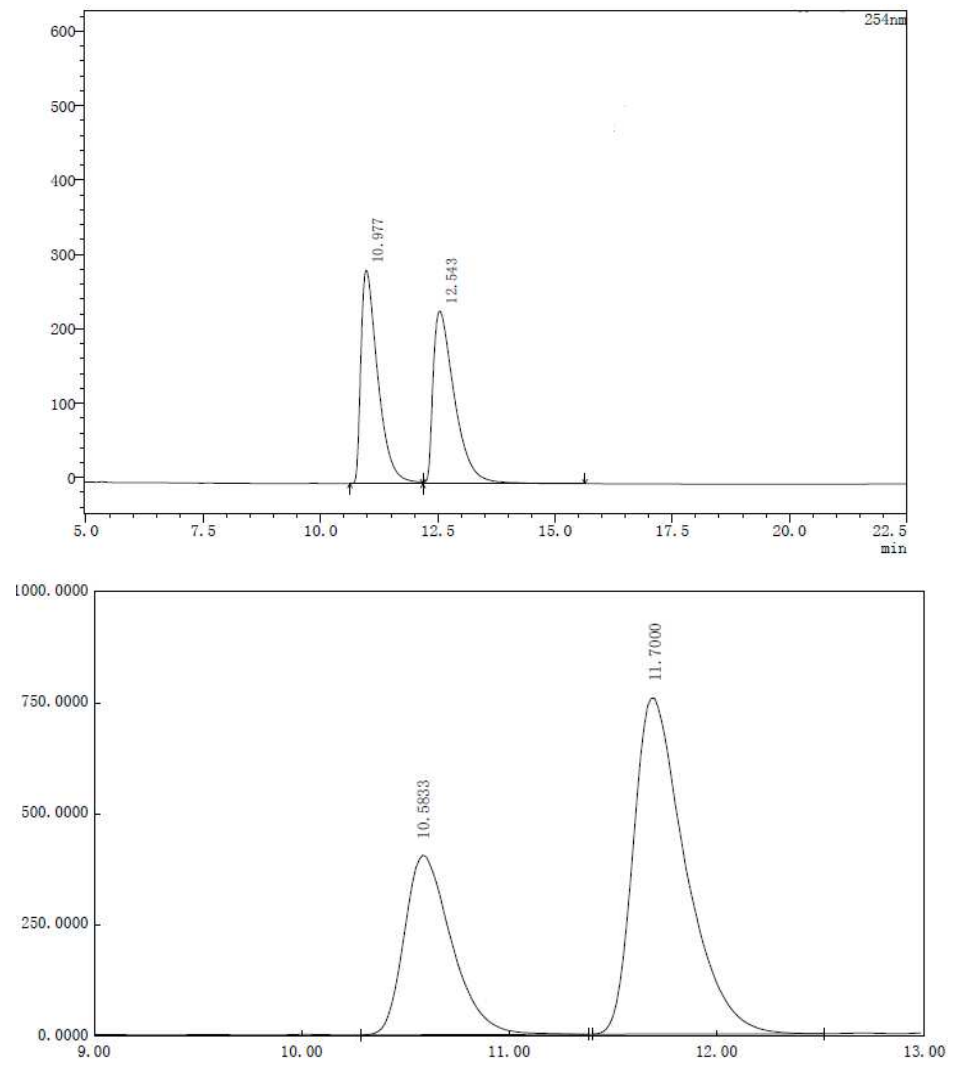

\begin{tabular}{|c|c|c|c|c|}
\hline Serial number & $\begin{array}{c}\text { Retention Time } \\
{[\mathrm{min}]}\end{array}$ & Area [mAbs*s] & Type & Area\% \\
\hline 1 & 10.5833 & 6387.2817 & BB & 32.6113 \\
\hline 2 & 11.7000 & 13198.8324 & BB & 67.3887 \\
\hline The Total & & 19586.1141 & & \\
\hline
\end{tabular}


<smiles>CC(C)S(=O)c1ccccc1</smiles>

Optical purity is determined by HPLC with a chiralcel OD-H column (hexane/i-PrOH $=93 / 7,0.5 \mathrm{~mL} / \mathrm{min}), \mathrm{t}_{\text {minor }}=18.12 \mathrm{~min}, \mathrm{t}_{\text {major }}=22.00 \mathrm{~min}$; ee $=12.4 \%$.
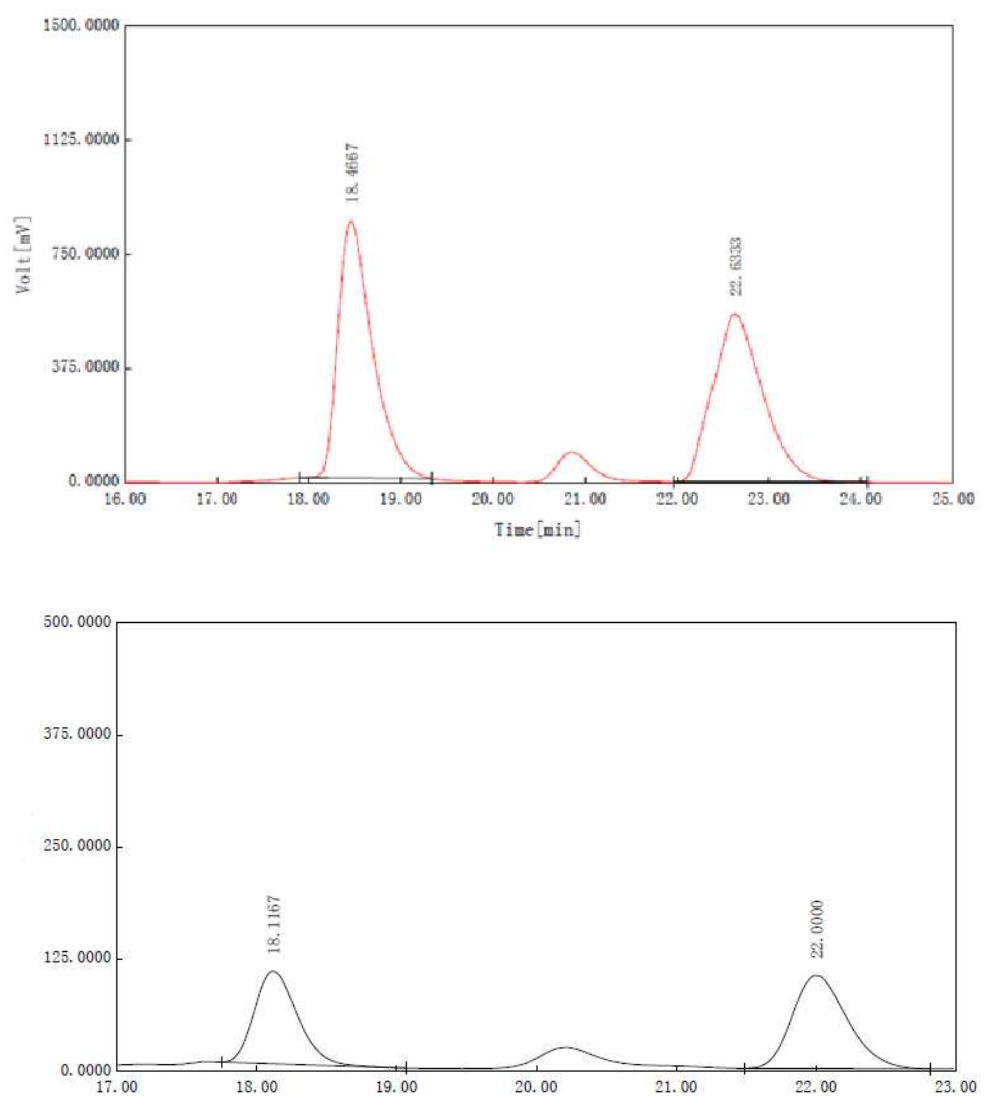

\begin{tabular}{|c|c|c|c|c|}
\hline Serial number & $\begin{array}{c}\text { Retention Time } \\
{[\mathrm{min}]}\end{array}$ & Area [mAbs*s] & Type & Area\% \\
\hline 1 & 18.11 & 2199.9234 & BB & 43.7897 \\
\hline 2 & 22.00 & 2823.9161 & BB & 56.2103 \\
\hline The Total & & 5023.8395 & & \\
\hline
\end{tabular}


<smiles>CS(=O)c1ccc(F)cc1</smiles>

Optical purity is determined by HPLC with a chiralcel OD-H column (hexane/i-PrOH $=90 / 10,1.0 \mathrm{~mL} / \mathrm{min}), \mathrm{t}_{\text {minor }}=19.21 \mathrm{~min}, \mathrm{t}_{\text {major }}=21.28 \mathrm{~min}$; ee $=21.8 \%$
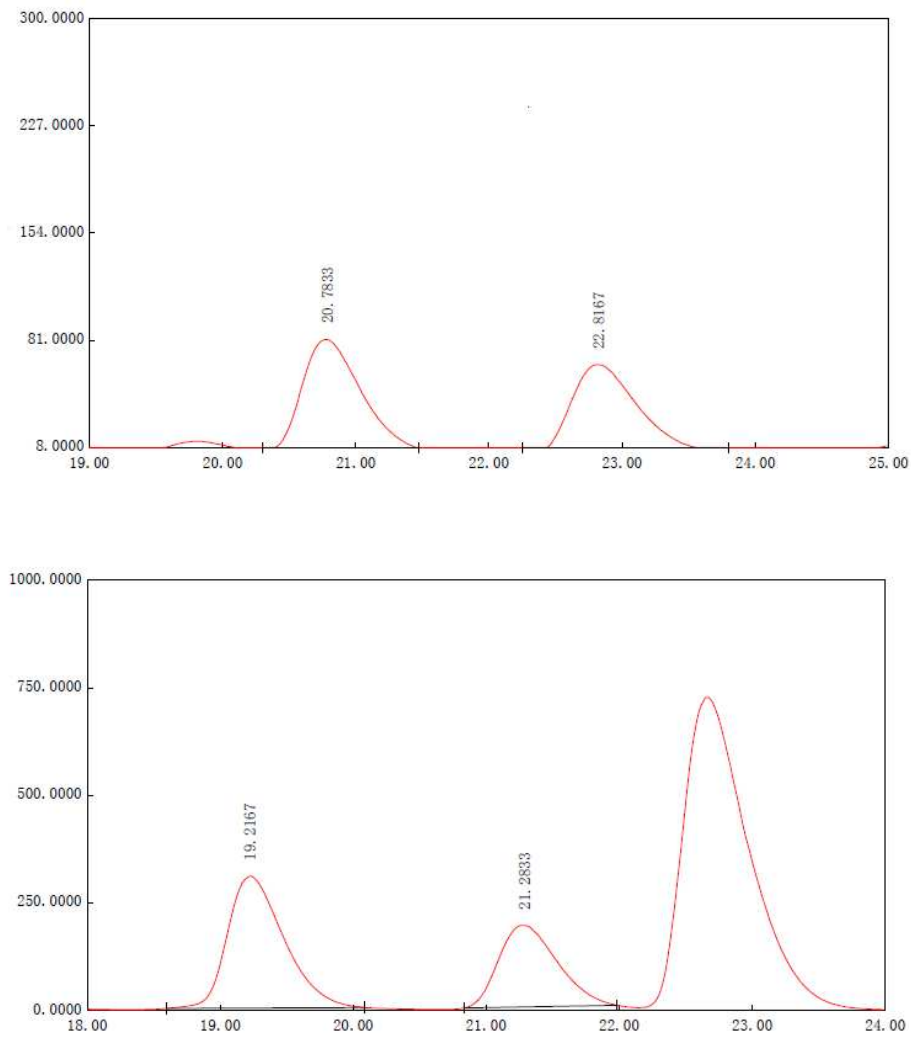

\begin{tabular}{|c|c|c|c|c|}
\hline Serial number & $\begin{array}{c}\text { Retention Time } \\
{[\mathrm{min}]}\end{array}$ & Area [mAbs*s] & Type & Area\% \\
\hline 1 & 19.2167 & 9017.6325 & BB & 60.8965 \\
\hline 2 & 21.2833 & 5790.4902 & BB & 39.1035 \\
\hline The Total & & 14808.1227 & & \\
\hline
\end{tabular}


<smiles>CS(=O)c1cccc(F)c1</smiles>

Optical purity is determined by HPLC with a chiralcel OD-H column (hexane/i-PrOH $=90 / 10,1.0 \mathrm{~mL} / \mathrm{min}), \mathrm{t}_{\text {minor }}=10.8 \mathrm{~min}, \mathrm{t}_{\text {major }}=11.9 \mathrm{~min} ; \mathrm{ee}=16.99 \%$.
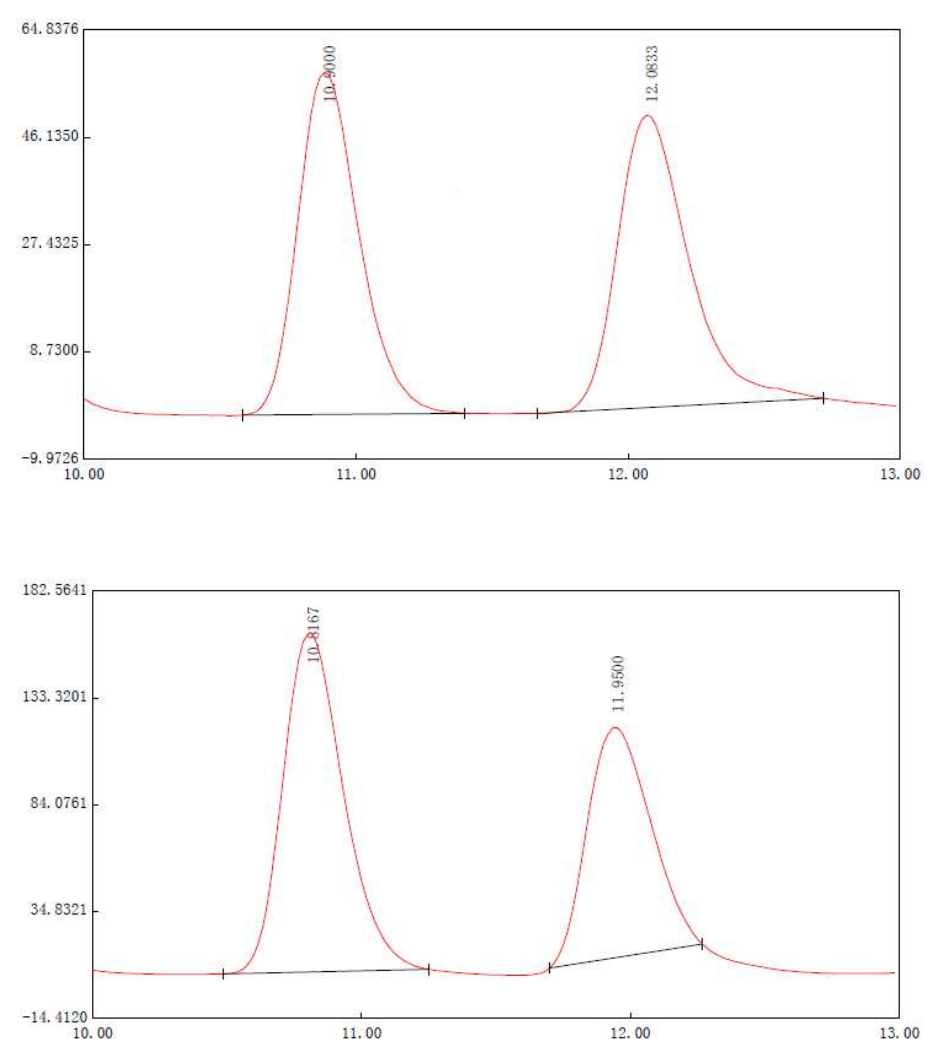

\begin{tabular}{|c|c|c|c|c|}
\hline Serial number & $\begin{array}{c}\text { Retention Time } \\
{[\mathrm{min}]}\end{array}$ & Area [mAbs*s] & Type & Area\% \\
\hline 1 & 10.8167 & 2550.4280 & BB & 58.4962 \\
\hline 2 & 11.9500 & 1809.5599 & BB & 41.5038 \\
\hline The Total & & 4359.9879 & & \\
\hline
\end{tabular}


<smiles>COc1cccc(S(C)=O)c1</smiles>

Optical purity is determined by HPLC with a chiralcel OD-H column (hexane/i-PrOH $=90 / 10,1.0 \mathrm{~mL} / \mathrm{min}), \mathrm{t}_{\text {minor }}=14.05 \mathrm{~min}, \mathrm{t}_{\text {major }}=18.60 \mathrm{~min}$; ee $=35.99 \%$.
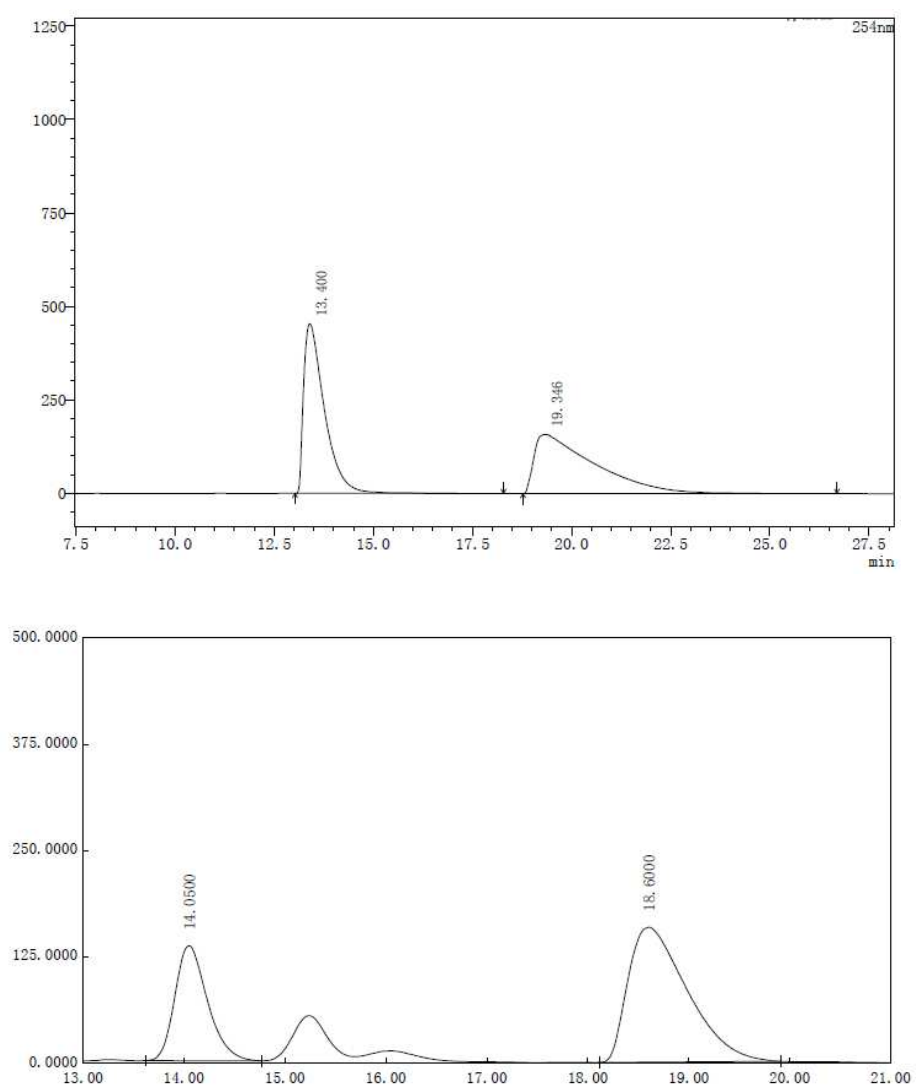

\begin{tabular}{|c|c|c|c|c|}
\hline Serial number & $\begin{array}{c}\text { Retention Time } \\
{[\mathrm{min}]}\end{array}$ & Area [mAbs*s] & Type & Area\% \\
\hline 1 & 14.0500 & 3031.3001 & BB & 32.0028 \\
\hline 2 & 18.6000 & 6440.6780 & BB & 67.9972 \\
\hline The Total & & 9471.9781 & & \\
\hline
\end{tabular}


<smiles>COc1ccc(S(C)=O)cc1</smiles>
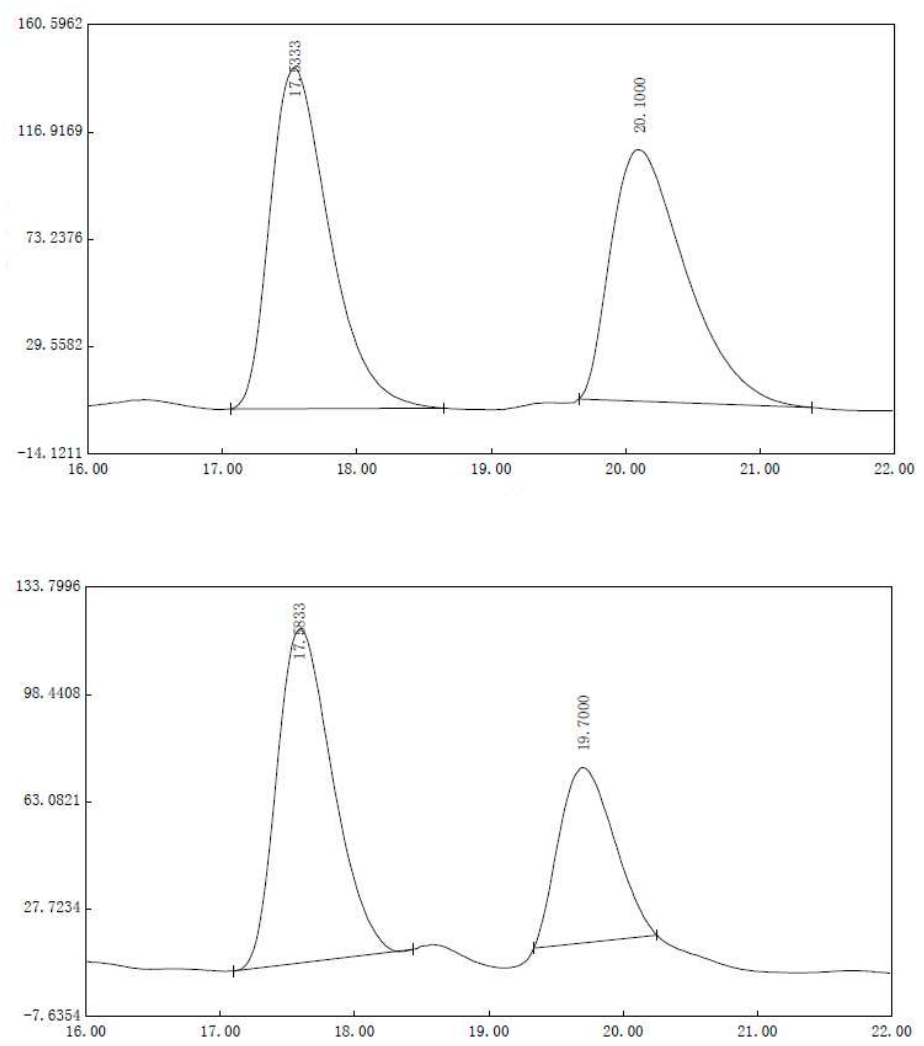

\begin{tabular}{|c|c|c|c|c|}
\hline Serial number & $\begin{array}{c}\text { Retention Time } \\
{[\mathrm{min}]}\end{array}$ & Area [mAbs*s] & Type & Area\% \\
\hline 1 & 17.5833 & 3306.3642 & BB & 65.8080 \\
\hline 2 & 19.7000 & 1717.8974 & BB & 34.1920 \\
\hline The Total & & 5024.2615 & & \\
\hline
\end{tabular}


<smiles>CS(=O)c1ccc(Cl)cc1</smiles>

Optical purity is determined by HPLC with a chiralcel OD-H column (hexane/i-PrOH $=90 / 10,1.0 \mathrm{~mL} / \mathrm{min}), \mathrm{t}_{\text {minor }}=20.30 \mathrm{~min}, \mathrm{t}_{\text {major }}=21.40 \mathrm{~min} ; \mathrm{ee}=25.1 \%$.
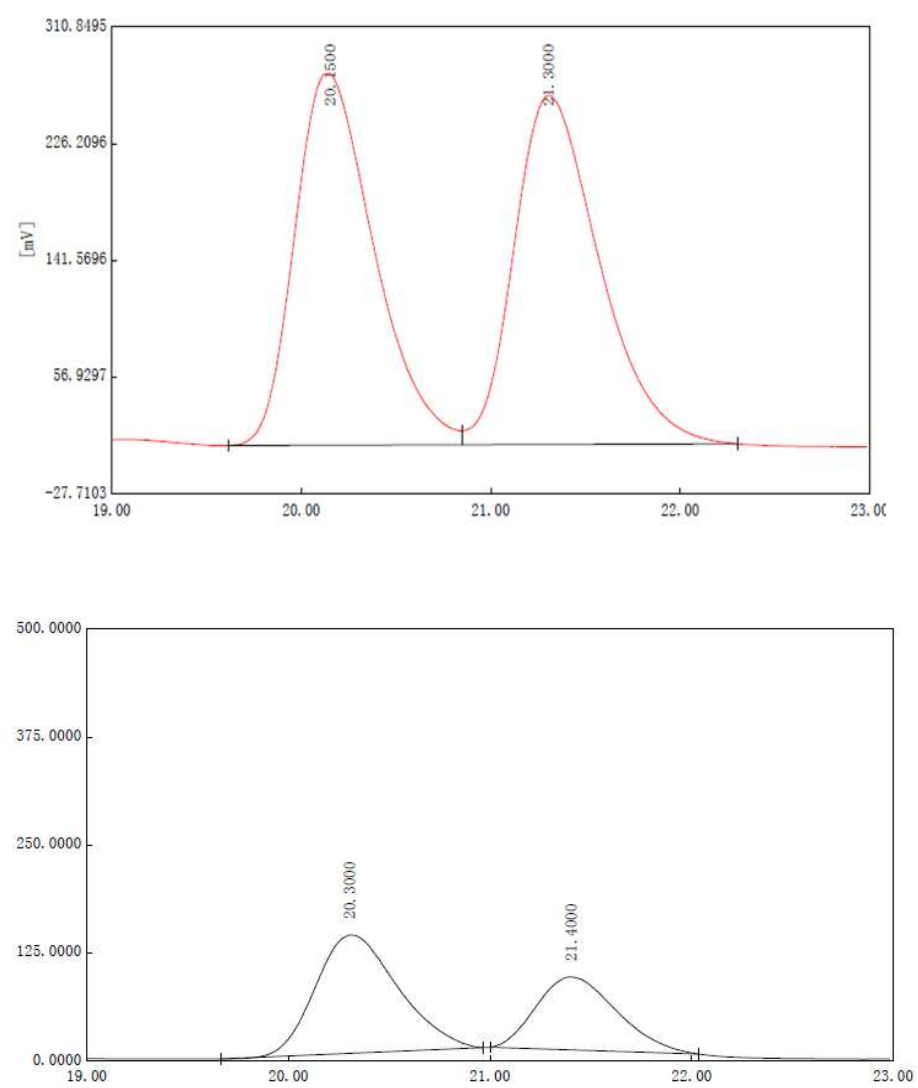

\begin{tabular}{|c|c|c|c|c|}
\hline Serial number & $\begin{array}{c}\text { Retention Time } \\
{[\mathrm{min}]}\end{array}$ & Area [mAbs*s] & Type & Area\% \\
\hline 1 & 20.3000 & 3884.8665 & BB & 62.4309 \\
\hline 2 & 21.4000 & 2337.8011 & BB & 37.5691 \\
\hline The Total & & 6222.6676 & & \\
\hline
\end{tabular}




\subsection{Recycle Experiments}<smiles>CS(=O)c1ccccc1</smiles>

Optical purity is determined by a chiralcel OD-H column (hexane/i-PrOH $=93 / 7,1.0$ $\mathrm{mL} / \mathrm{min}$ )

\begin{tabular}{|c|c|}
\hline Run & ee(\%) \\
\hline 1 & 47 \\
\hline 2 & 43.5 \\
\hline 3 & 41.2 \\
\hline
\end{tabular}

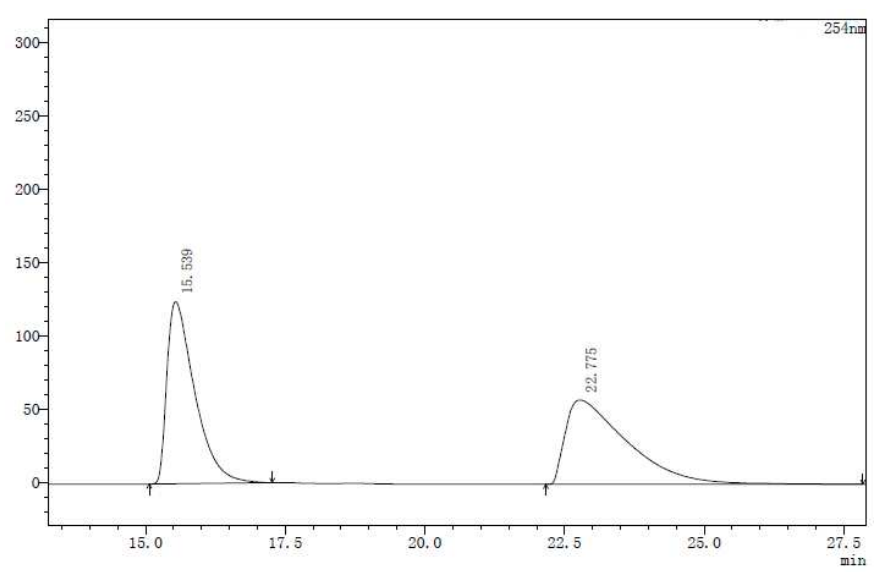

\section{Run 1}

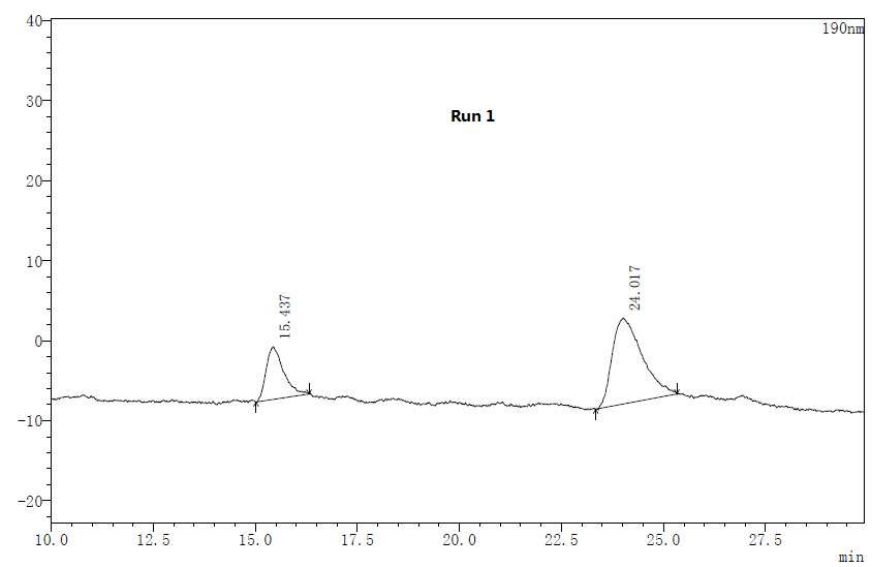

\begin{tabular}{|c|c|c|c|c|}
\hline Serial number & $\begin{array}{c}\text { Retention Time } \\
{[\mathrm{min}]}\end{array}$ & Area [mAbs*s] & Type & Area\% \\
\hline 1 & 15.437 & 196104 & FF & 26.467 \\
\hline 2 & 24.017 & 544832 & FF & 73.533 \\
\hline The Total & & 740936 & & ee $=47 \%$ \\
\hline
\end{tabular}




\section{Run 2}

wV

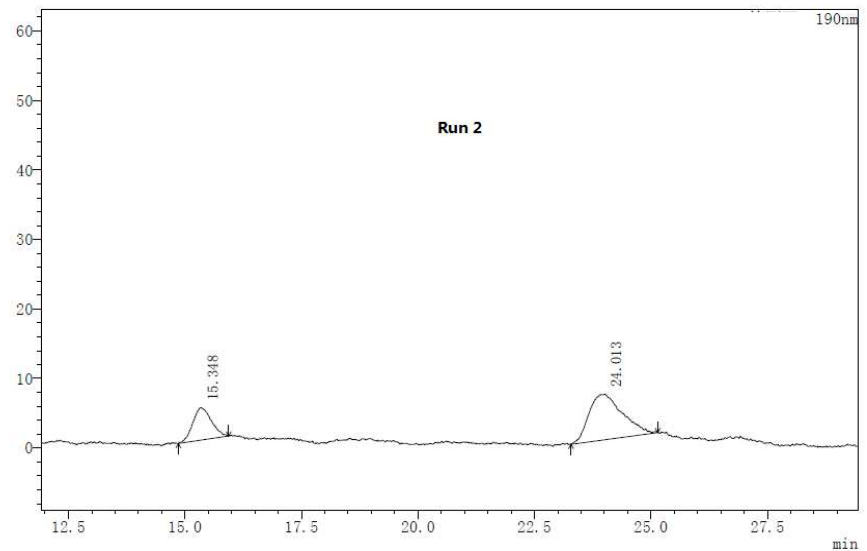

\begin{tabular}{|c|c|c|c|c|}
\hline Serial number & $\begin{array}{c}\text { Retention Time } \\
{[\mathrm{min}]}\end{array}$ & Area [mAbs*s] & Type & Area\% \\
\hline 1 & 15.348 & 129540 & FF & 28.220 \\
\hline 2 & 24.013 & 329502 & FF & 71.780 \\
\hline The Total & & 459042 & & ee $=43.5 \%$ \\
\hline
\end{tabular}

\section{Run 3}

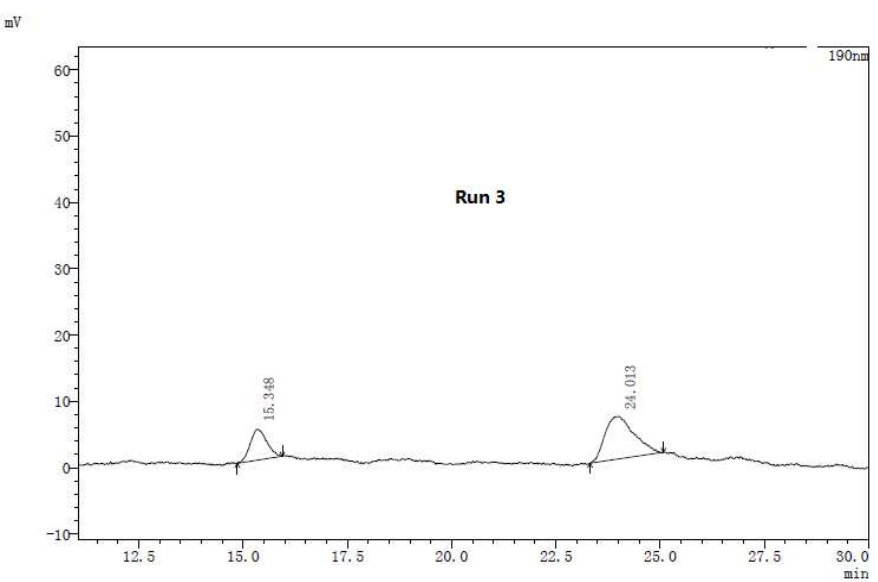

\begin{tabular}{|c|c|c|c|c|}
\hline Serial number & $\begin{array}{c}\text { Retention Time } \\
{[\mathrm{min}]}\end{array}$ & Area [mAbs*s] & Type & Area\% \\
\hline 1 & 15.348 & 129540 & FF & 29.412 \\
\hline 2 & 24.013 & 3190896 & FF & 70.588 \\
\hline The Total & & 440436 & & ee $=41.2 \%$ \\
\hline
\end{tabular}




\section{Kinetics of adsorption}

$6.8 \mathrm{mg}$ racemic (methylsulfinyl)benzene dissolved in $0.6 \mathrm{~mL} \mathrm{CD}_{3} \mathrm{OD}$ was placed in a NMR tube and got the ${ }^{1} \mathrm{HNMR}$ noted blank ( $\left.0 \mathrm{~min}\right)$; then $30 \mathrm{mg}$ evacuated 1 was added into the tube stayed at $-10{ }^{\circ} \mathrm{C}$ and determined the ${ }^{1} \mathrm{HNMR}$ at a certain time. From the spectra, we found that after $2.5 \mathrm{~h}$ the adsorption reached equilibrium comparing theratio of $\mathrm{CH}_{3}$ (from (methylsulfinyl)benzene)and $\mathrm{H}_{2} \mathrm{O}$ (from $\mathrm{CD}_{3} \mathrm{OD}$ ) at different time, We calculated the ratio between the (methylsulfinyl)benzeneand 1 is approximately 1:1 (the adsorption of the water and methanol is out of consideration).

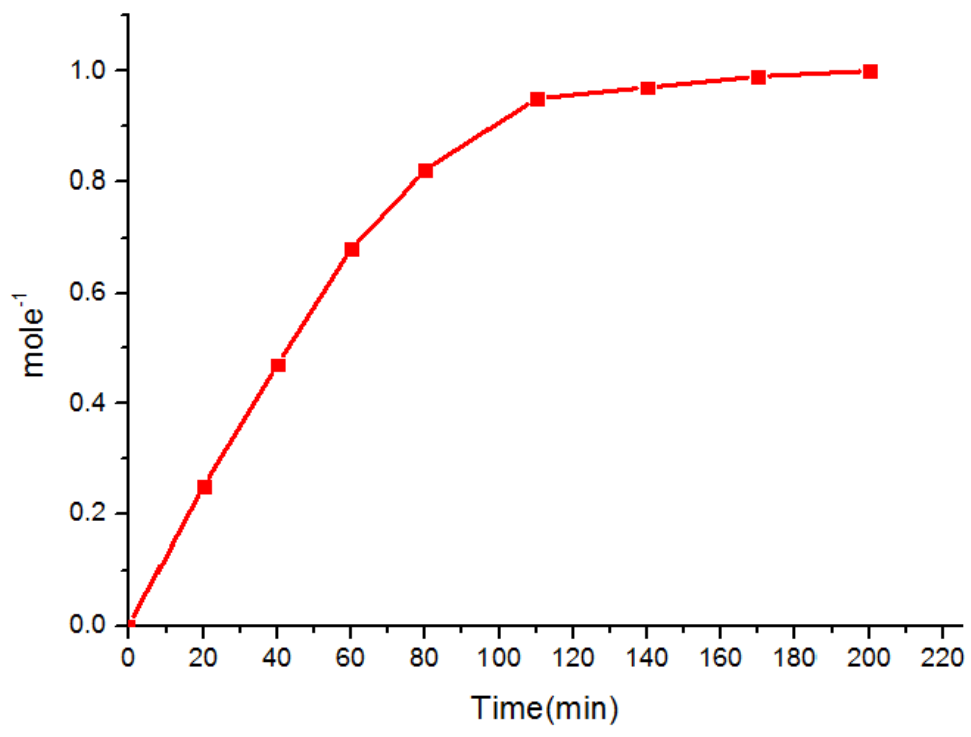

\section{Figure S14. FT-IR spectra}

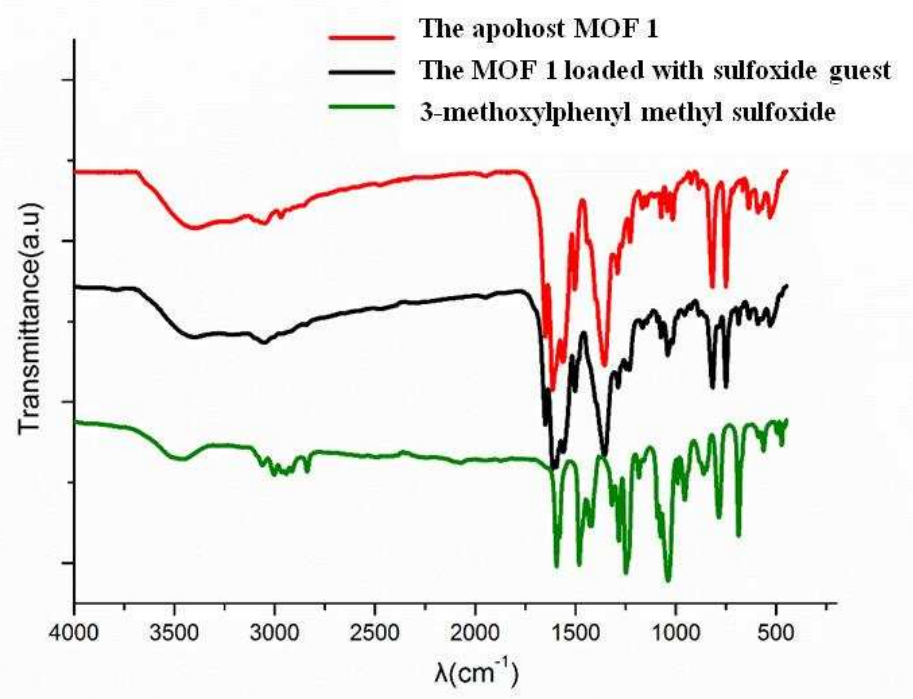

